\title{
Identification and analysis of Swiss National Road tunnels pathologies
}

Federica Sandrone, Vincent Labiouse

Rock Mechanics Laboratory (LMR)

Ecole Polytechnique Fédérale de Lausanne (EPFL), Switzerland

This is the accepted author's version of an article protected by copyright.

The rights are held by Elsevier.

The final publication is available at

http://www.journals.elsevier.com/tunnelling-and-underground-space-technology

Article published in:

Tunnelling and Underground Space Technology (ISSN 0886-7798)

Volume 26, Issue 2, March 2011, Pages 374-390

Digital Object Identifier (DOI): 10.1016/j.tust.2010.11.008 


\section{Identification and analysis of Swiss National Road tunnels pathologies}

F. Sandrone* and V. Labiouse

Rock Mechanics Laboratory LMR, Ecole Polytechnique Fédérale de Lausanne EPFL, Switzerland

\section{ABSTRACT:}

In order to evaluate the general conditions (i.e. safety, serviceability and durability) of tunnels, a detailed analysis of the lining structure and surrounding rock mass is required. During the service life of the tunnel, regular survey activities should be performed to check the tunnel conditions and regular conservation practices are necessary to preserve the tunnel's structural integrity and guarantee safety and serviceability during operation. The collection of data about tunnels since their construction and during their service life may help in the assessment of tunnel conditions and improve the understanding of tunnel degradation with time. The long term behaviour of the Swiss National Road tunnels has been studied and a specific data base has been developed. Based on detailed literature review and analysis of principal inspections results collected in the data base, the main pathologies that affect the Swiss road tunnels have been identified. In addition, by means of a specific data analysis, both degradation potential and rate have been investigated and the main influence factors involved have been selected. The obtained results confirm what was expected according to observations in the past. After a brief introduction about the tunnels of the National Roads network, this paper describes the main steps of the data analysis, different methods used and main results of the analysis. Due to the fact that the tunnels within Swiss National Roads were developed during the sixties, attention has been focused only on concrete lined tunnels.

KEYWORDS:

Road Tunnels pathologies, Degradation, Data Base, Correspondence Analysis, G.I.S. tools, Maintenance

\section{INTRODUCTION}

During the service life of a tunnel, like any structure, safe and effective operation should be ensured. The tunnel must fulfil its design purposes without creating circulation restraints to normal users or safety problems. Underground constructions have longer service life when compared to other structures and several factors such as material quality and weathering actions may contribute to reduce their durability. The distinctive feature of a tunnel is the direct interaction between the lining and the rock mass. Load conditions may vary significantly with time due to changes in rock mass behaviour and environmental 
actions. Various chemical, physical and mechanical processes may determine the deterioration of exposed surfaces of the rock mass and the lining. It is possible to distinguish between:

- the chemical action, properly named weathering, caused by the chemical interaction of the tunnel with the surrounding environment;

- the physical action, ageing, ruled by time dependent changes affecting the characteristics of concrete lining and rock mass;

- the other actions, mainly mechanical, such as pore water pressure redistribution, ice and frost, fires and car collisions.

All these processes may change the tunnel equilibrium conditions with time (Sandrone and Labiouse, 2010) and contribute to the changes in tunnel serviceability, stability and safety conditions.

During operation, the tunnel owner should perform regular preventive maintenance to avoid major deficiencies and reduce pathologies development. Tunnel pathologies may appear in the form of several disorders depending on the building materials (e.g. quality and porosity), construction techniques and operation conditions. Following disorders evolution (e.g. cracks, deformations and water damages), regular inspections may reveal time dependent pathologies of the lining and/or the rock mass and allows the owner to check the structural and general conditions of the tunnel.

As shown in Figure 1, if the tunnel does not fail due to a sudden problem, caused by external events (e.g. earthquake, fire) or by important structural deficiencies at construction, its quality level gradually deteriorates. Routine maintenance does not improve the quality level of the whole structure but it is necessary to guarantee the normal operation by slowing (or prevent in the case of preventive maintenance) the deterioration process. When rehabilitation and repair are carried out to cope with major problems, the result is a rise of quality level with an extended service life. If no conservation activity is performed, the tunnel quality level will reach a minimum value (i.e. serviceability limit state) and it becomes necessary to perform urgent rehabilitation and repair to prevent the quality level from reaching the ultimate limit state, which means that the tunnel does not ensure safe operation any more. In that case, both structural and users safeties are no longer ensured and a complete tunnel refurbishment is required. In other cases, at the end of the tunnel estimated service life, a complete tunnel renewal is necessary in order to extend operation, for example by adapting the tunnel to new operational, safety and/or ventilation requirements.

\section{Fig. 1}


The goals of conservation procedures for tunnels are:

- Maintain the regular functions, in terms of both serviceability and safety, of the structure.

- Preserve the tunnel characteristics to guarantee its future life span and economical value,

- Estimate the tunnel performance in the future.

In order to achieve these goals, the tunnel owner should regularly perform technical survey of the structure and decide on the ordinary procedures of maintenance and/or major repairs. Effective tunnel conservation depends on correct diagnoses of pathologies origins and deterioration processes. Starting from specific initial conditions, defined by construction data, tunnel durability can be estimated by considering the degradation processes, which are dependent on environmental and operational conditions as well as on conservation activities.

Initial conditions such as construction method, geological and hydrogeological conditions determine the degradation potential of a structure and the tunnel operation environment and conditions (e.g. traffic, ventilation system) influence the degradation rate. Tunnel inspections are mainly focused on the visual aspect of the tunnel intrados, condition of concrete lining conditions in this case. The main causes of detected tunnel disorders for several countries, based on a detailed literature review about inspection results, are compiled and as shown in Table 1. Though geological and hydrogeological conditions change from country to country, the interactions between the tunnel and the groundwater (which significantly depends on waterproofing and drainage systems) and between the tunnel and the surrounding ground are considered the principal causes for tunnels deterioration. Tunnel environment also plays an important role in lining degradation. In particular, temperature, humidity, ice and frost are mentioned as principal causes of tunnels deterioration in almost all the countries considered in the literature review. Other identified causes are related to tunnel operation including aggressive atmosphere, which are dependent on traffic pollution and ventilation system, and use of de-icing salts and the associated corrosive actions.

Table 1

Supported by the Swiss Federal Roads Authority, the Rock Mechanics Laboratory of the Ecole Polytechnique Fédérale de Lausanne (Switzerland) created and compiled a Data Base for National Roads tunnels. Based on this data collection, after a general portrait of Swiss National Roads tunnels, tunnel degradation potential and rate have been determined following a specific data analysis process. In the first part of this paper tunnels are described in terms of their degradation potential determined by considering the initial conditions. Then, based on principal inspections results, the paper focuses on the degradation 
rate which characterises the tunnels during their service life. The main factors that determine disorders development are pointed out using a multivariate statistical approach. Finally, representative pathology scenarios are identified and described.

\section{SWISS ROAD TUNNELS}

\subsection{Tunnel Data Base}

The development of the Swiss National Road network began 1960s. The network comprises a fairly large number of tunnels and all tunnels have a concrete lining. Some tunnels have been in operations for more than 40 years and need maintenance to ensure everyday serviceability and safety. In 2006 tunnels, the conservation costs were almost doubled when compared to the costs four years ago (OFROU, 2006). In Switzerland, the Swiss Federal Roads Authority has a data base called KUBA-DB (OFROU, 2004) and it is used to manage the road structures. KUBA-DB, in its actual form, is not convenient for collecting data about tunnels and it is necessary to create a specific data base for tunnels to have enough information for studying the long term behaviour of road tunnels.

By analysing available literature about road tunnels degradation and conservation procedures (Széchy, 1966; Richards, 1998; Iftimie, 2001; CETu, 2004) together with the directives of several countries (see Table 1; Richards, 1998; Fed. Highway Admin. and Fed. Transit Admin., 2003a and 2003b; Highways Agency et al., 2003) and advices from tunnel inspectors (people from CETu - France and PERSS Ingénieurs conseils SA Fribourg, Switzerland), it has been possible to outline the main features that characterise an appropriate tool for managing information about structures belonging to transportation networks and to create a specific data base for road tunnels. The Swiss Tunnel Data Base (Sandrone et al., 2007a) regroups information of different type and nature, related to the whole tunnel life and necessary for describing the actual conditions of the structure, in particular (Table 2):

- Construction data (e.g. construction method, lining material and quality, drainage and waterproofing systems, etc.) together with geological and hydrogeological information,

- Information from tunnel inspections, about the main disorders that affect road tunnels, together with detailed data about operative environment (traffic, ventilation) and maintenance procedures (type, frequency).

Table 2 
The Swiss Tunnel Data Base was created using commercial software for data collection and treatment (Access - Microsoft Office Professional ed. 2003). An important feature that characterises the TDB is the heterogeneity of collected information. Both qualitative (e.g. pathology description, geology, etc.) and quantitative (e.g. structural and geometrical data) data are used for describing each tunnel. Some information stored in the data base was compiled directly from tunnel inspector's reports, which may be very different from canton to canton and required adjustments before being analysed. In order to maximise data consistency, several attributes (modalities) for each factor (variable) have been identified and coded.

The Swiss Tunnel Data Base, at present, stores detailed information about 168 tunnels (Figure 2). Only few cantonal administrations contributed to the data collection by filling a specifically developed technical form. The majority of data was collected directly by LMR-EPFL people (F. Sandrone and J.-F. Mathier) by consulting cantonal archives and specific literature.

\section{Fig. 2}

\subsection{National Road tunnels portrait}

Using the information stored in the data base, it is possible to compile a portrait of the Swiss National Roads tunnels. Pie charts in Figure 3 and Figure 4 describe the main qualitative features characterising the Swiss National Roads tunnels. For each selected feature, statistics show how the whole data base population (i.e. [TDB]) is distributed according to feature modalities. These statistics also indicate the distribution of the 122 “inspected tunnels” (among the 168 of the TDB) for which principal inspection data have been collected and compiled in the data base [PI].

\section{Fig. 3}

First, tunnels can be characterised in terms of age and overburden (Figure 3). The age of the tunnel is calculated in terms of service life duration, starting from the commissioning year (operation), which means considering operation as an active cause of tunnel degradation. The Swiss Road Tunnels can be divided into 3 main classes (Sandrone et al., 2007a):

A. Old tunnels: constructed before the year 1970. Operating for about 40 years, this class of tunnels represents the $19 \%$ of the population of the data base.

B. Rather old tunnels: excavated between the years 1970 and 1990. This class represents about the 43\% of the total population. 
C. Recent tunnels: constructed after the year 1990. Operating for less than 20 years, this class of tunnels represents about the $38 \%$ of the total population of the data base.

For each age class it has been evaluated, according to the collected data, the percentage of tunnels that has been inspected. The majority of tunnels constructed and commissioned before the nineties has been inspected in recent years (i.e. 13 tunnels of class A and 28 tunnels of class B were inspected between 2005 and 2006). However, no information was found on the last principal inspection for some tunnels, especially the recent ones (i.e. about 35 tunnels commissioned after the year 1990).

Considering that the maximum depth for many Swiss National Roads tunnels is rather similar to the mean depth and in case of short tunnels, which the whole tunnel is usually designed for bearing the highest load, tunnels have been classified according to their maximum depth into three main classes: low overburden (LO), medium overburden (MO), high overburden (HO). For about 20 tunnels, which the depth information was lacking in the technical form, the data has been retrieved by superposing tunnel position and Swiss 1:25000 Digital Terrain Model (source: SWISSTOPO - MNT25) with a G.I.S. As in Figure 3, most of the tunnels are characterised by a low to medium overburden while tunnels with a high overburden represent only $16 \%$ of the population of the data base.

G.I.S. geotechnical data were used for supplying the lack of geological and hydrogeological data available in the documentation found in cantonal archives (Sandrone et al., 2007b). Apart from quaternary deposits (i.e. Loose Ground, LG), the geotechnical classes of the Swiss 1:200000 vector map (source: OFEV - Carte géotechnique simplifiée de la Suisse) were merged in five principal classes:

- $\quad$ Clays and Marls (C\&M),

- Carbonates (Ca)

- Evaporites (Ev),

- Schists and Phyllites (Sc\&Ph),

- Crystalline silicate rock masses and gneiss (Cry).

For tunnels that cross more than one type of rock mass, a contribution proportional to the excavated length in each class has been considered. The geological distribution of the tunnel population is shown in the geology pie chart (Figure 4).

\section{Fig. 4}

Geological conditions and year of construction determine the construction method and other tunnel features (e.g. shape, size, type and quality of building materials). As presented in Figure 4, the majority of 
tunnels (i.e. 67.5\%) have been excavated with the traditional drill and blast method. Full face excavation is used more recently and characterises about $17 \%$ of the data base population (considering TBM and Shield together).

Again, for lack of hydrogeological data in the tunnel documentation, the Swiss hydrological vector map (source: Swiss 1:200000 hydrological map - OFEV) has been used for classifying tunnels in terms of water circulation in the surrounding rock mass (the terminology used by the OFEV is also kept in this context):

- about $39 \%$ of tunnels crosses discontinuous formations with water circulation through discontinuities (i.e. fissured as crystalline and gneiss rock masses and partly fissured porous rock masses as schists and phyllites, with water circulation through discontinuities),

- about $24 \%$ of tunnels crosses porous formations as quaternary deposits, marls and clayey media,

- $37 \%$ of tunnels crosses karstified formations as carbonates and evaporites (gypsum and anhydrite formations),

Except for a small number of old tunnels without waterproofing system (i.e. no waterproofing, No), tunnel are characterised by the internal waterproofing system placed at the definitive lining intrados (i.e. tunnel intrados, I). It is possible to identify two main types of waterproofing in the Swiss National Roads tunnels (Figure 4):

- the partial system (i.e. crown and side walls) is typical of traditional excavation techniques. In majority of the tunnels, it is placed between the support and the definitive lining (i.e. partial in the middle, Pm), but it can also be placed in direct contact with the excavated rock mass (i.e. partial extrados, Pe)

- the total system (i.e. crown, side walls and invert) is mainly associated with circular section and full face mechanical excavation. Similar to the partial system, for majority of cases, it is placed between the support and definitive lining (i.e. total in the middle, Tm). In few cases, it is placed in direct contact with the rock mass (i.e. total extrados, Te). In the case of segmental lining, the waterproofing between the segments has been considered as total in the middle (Tm).

With regard to operation conditions, traffic and ventilation system are represented in Figure 4. According to (SN 640 908, 2000), it is possible to categorise tunnels into four classes based on the Average Daily Traffic Volume (i.e. DTV, data for the year 2006 from OFROU): 
- $12 \%$ of the total population of the data base is characterised by low traffic: $D T V \leq 10$ ' 000 vehicles;

- $42 \%$ is characterised by a medium traffic: $D T V=10^{\prime} 000 \div 30$ ' 000 vehicles;

- more than $18 \%$ has high traffic conditions: $D T V=30$ ' $000 \div 50$ ' 000 vehicles;

- $9 \%$ is crossed daily by a very high traffic: $D T V>50$ ' 000 vehicles;

- for the last $19 \%$ (i.e. 32 tunnels) no data is available.

Except about $40 \%$ of the tunnels population that is characterised by a natural ventilation, the majority of structures is equipped with a longitudinal ventilation system (more than $48 \%$ ) and the rest is equally divided between transverse and semi-transverse systems (see Figure 4). As ventilation system mainly depends on tunnel length and daily traffic, their relationship has been investigated. Short tunnels (i.e. less than 700-800 m long) are characterised by the absence of a ventilation system (i.e. natural ventilation) or by longitudinal ventilation, in the case of high and very high traffic. Tunnels of medium length (i.e. between $800 \mathrm{~m}$ and $2000 \mathrm{~m}$ long) generally have longitudinal ventilation system. For few cases with very high traffic conditions, tunnels have transverse ventilation. Long tunnels (i.e. more than $2000 \mathrm{~m}$ long) have either transverse or semi-transverse ventilation.

\subsection{Main disorders}

Based on the detailed literature review presented in the introduction and on the information collected by tunnels inspectors, 17 typical tunnel disorders were identified and documented in the Tunnel Data Base. They are listed in Table 3, together with the number of records and affected tunnels (with reference to the last principal inspection). Many disorders are typical of concrete structures (e.g. efflorescence, calcium leaching, de-icing salts corrosion, etc), as the tunnels in the national road network developed from 1960s are characterised by a concrete or, in some cases, a reinforced concrete lining. Concrete lining degradation due to Alkali-Aggregate Reaction (AAR) is not reported among disorders because Swiss tunnels inspectors have just begin to be aware about this problem. This phenomenon has only been identified in Switzerland on several types of structures, in particular dams and bridges, in recent years. The AAR pathology may indeed take more than 20 years before becoming visible (Merz and Hammerschlag, 2000) and at present, it is still quite difficult to recognise its symptoms by means of simple visual inspections. Note that disorders affecting the electromechanical equipment were not considered in the analysis. 
As neither voids behind the lining [7] nor local wall deformations [10] have been recorded during the visual inspections and, local crown deformations [9] have only been observed in one tunnel and cannot be considered as representative, only 14 disorders will be considered for the analysis.

\section{DATA ANALYSIS}

The tunnel features considered in the following analysis process will be referred to as influence factors. Each of them is characterised by several modalities/attributes. For example, the average daily traffic volume (DTV) is an influence factor characterised by 4 different modalities: low, medium, high, and very high. The geological conditions, another influence factor, is characterised by 6 modalities which correspond to the typical geological formations presented in Section 2.2 (Figure 4, i.e. loose ground, clay and marls, carbonates, evaporites, schists and phyllites, and crystalline silicate rock masses and gneiss). According to the description of the data base presented in Section 2, it is clear that the high number of variables (i.e. number of disorders $\times$ number of influence factors, each of them $\times$ number of attributes/modalities) could be analysed only by using specific exploratory data analysis techniques. The whole analysis process is summarised in Figure 5:

1. Evaluation of initial conditions influence on tunnel degradation potential. In this first analysis step (see Section 3.1) the TDB is analysed through frequency tables (i.e. contingency tables) for evaluating the distribution of categorical variables (i.e. modalities) in the recorded sample. Though at a very general level, this method allows pointing out evident and/or systematic pathology development scenarios related to tunnel initial conditions (i.e. construction features, geology and other characteristics that depend on tunnel geographical location, as depth, for example) and which define the tunnel degradation potential.

2. Evaluation of dependencies between identified pathologies (i.e. disorder type) and the different features that characterise tunnel service life. In the second analysis step (see Section 3.2) the influence of tunnel service life/operation conditions on the degradation speed/rate is investigated by means of multivariate statistics tools (i.e. Correspondence Analysis).

3. Finally, the disorder probability due to one or more influence factors modalities/attributes is evaluated. This third step (see Section 3.3) is necessary to understand whether (or not) factors modalities identified in the previous steps have a major influence on the selected disorder development. Moreover, by taking into account the number of affected tunnels and the interactions between the 
selected influence factors, it is possible to better describe representative scenarios for disorders development.

Since the number of tunnels for which it was possible to collect inspection results does not correspond to the total population of the TDB (i.e. 122 over 168), data analysis can be considered representative for about $73 \%$ of the whole data base population. In order to avoid data redundancy, due to successive inspections of the same tunnel (i.e. repeated observations of the same disorder, if any), the number of disorders has been calculated by taking into consideration only data from the last tunnel principal inspection.

\section{Fig. 5}

\subsection{Initial conditions: tunnel degradation potential}

The tunnel degradation potential has been evaluated by calculating the mean number of disorders per tunnel for each feature modality class. Tunnel degradation potential should be analysed by considering classes of tunnels with the same initial conditions. In order to avoid performing analyses on very few populated classes, due to the high variability of tunnel initial conditions, a preliminary selection of features has been operated for reducing the number of variables defining tunnel initial conditions.

\subsubsection{Construction features: commissioning year, construction method and waterproofing system}

As shown in Figure 6, the mean number of disorders per tunnel, recorded during the last principal inspection, is slightly higher for tunnels constructed before the year 1970, if compared with recent tunnels.

Considering the construction techniques the higher mean number of disorders characterises tunnels constructed under a shield protection and thus in critical geological conditions, followed by tunnels excavated by drill and blast and road-header. In the drill and blast technique, especially in the past, the use of explosives increased the size of the disturbed/damaged zone around the tunnel and increased deterioration due to weathering. Road-header is mainly used in medium resistant sedimentary rocks characterised by a high weathering potential (Sandrone et al., 2007b).

Another frequently observed problem is the high degradation potential that characterises tunnels without waterproofing system (No) or with an internal waterproofing (I). Figure 6 shows how several disorders may also affect tunnels with a total waterproofing system (Tm). Indeed, in the absence of an 
adequate drainage system, tunnel disorders may be caused by additional loads development due to water pressure increase around the tunnel.

\subsubsection{Geological and hydrogeological conditions}

Higher mean number of disorders characterises tunnels excavated in loose ground masses (LG), probably due to their poor quality and the low depth (weathered rocks). Tunnels excavated in evaporites (Ev) are affected by a relevant number of disorders mainly due to their swelling potential while tunnels in crystalline rocks (Cry), probably due to the fact that this formation in Switzerland is often rather blocky, are more sensible to groundwater weathering action. Time dependent (delayed) behaviour of rock masses may have a critical impact on tunnel degradation potential mainly by changing tunnel stability (e.g. external load increase vs. insufficient lining bearing capacity) as demonstrated by the rather significant number of disorders observed in schists and phyllites (Sc\&Ph), and in marl and clayey media (C\&M).

Considering the hydrogeological conditions around the tunnel, the contribution on tunnels degradation potential of water circulating in porous rock masses may be significant according to the terminology proposed by the OFEV this class groups quaternary deposits, marls and clayey media). As a matter of fact, in addition to the weathering action of the groundwater around the excavation, the redistribution of water pressures inside the rock mass and on the tunnel lining can induce significant delayed effects and a progressive change in the tunnel stability conditions (Gärber, 2003; Carranza-Torres and Zhao, 2009). The low degradation potential characterising karstic formations is essentially due to the fact that water circulates mainly through karst channels (about 95\% according to Worthington, 2003 and Filipponi, 2009), reducing the weathering action to a limited and defined zone. On the contrary, the weathering potential of discontinuous rock masses is higher since the water has not a well defined path to follow.

\section{Fig. 6}

\subsection{Operation conditions: tunnel degradation rate}

After analysed the tunnel degradation potential, the influence of service life conditions on the tunnel degradation rate has been investigated. Factors that influence degradation rate have been selected from all the features that characterise the tunnel during its service life. In terms of collected information, attention is focused on age, traffic volume, ventilation system, waterproofing system and also on rock mass behaviour and chemical composition of groundwater which mainly depends on the rock mass type. 
Section 3.2.1 investigates the influence of the tunnel operating environment on the lining degradation rate. Due to a lack of information on exhaust composition, temperature and humidity, traffic conditions and ventilation system were considered to evaluate the influence of the atmosphere quality inside the tunnel.

Section 3.2.2 analyse the influence of the geological and hydrogeological conditions on the degradation rate. Although these factors were already considered for evaluating the tunnel degradation potential, the surrounding rock mass and groundwater evolve with time are responsible for delayed effects and must be reconsidered.

As disorders are observed in several tunnels, it is quite complicated to find specific conditions of pathology development. However, by using appropriate analytic processes such as the Correspondence Analysis (Greenacree, 1984; Moreau et al., 2000; Nishisato, 2004), it is possible to find the most relevant dependencies between observed disorders and influence factors and to identify recurrent pathologies and main factors that determine tunnel degradation rate.

\subsubsection{Service life duration, operation and environment conditions}

As the operation environment of road tunnels obviously depends on traffic volume, a correspondence analysis was performed for identifying relationships between the average daily traffic volume (DTV data from year 2006) and observed disorders. For this purpose, data are resumed in several contingency tables of $n_{\text {rows }} \times m_{\text {columns. }}$ The number of columns $\left(m_{\text {columns }}\right)$ represents the problem dimension which corresponds to the influence factor modalities (in this case the 4 DTV classes described in Section 2.2, $m_{\text {columns }}=4$ ), while the number of rows represents the disorders $\left(n_{\text {rows }}=14\right)$. This multidimensional problem turns into a simpler 2-dimensional scatter plot (Figure 7) called bi-plot, where it is possible to represent together column points’ pattern and row points (i.e. represented respectively by lettered triangles and numbered dots). The distance between row points describes how disorders are similar to each others with regard to the influence factor modality. Moreover, the distance between column points and row points denotes the eventual dependency of the disorder on the influence factor attribute. As it is possible to observe on the right-hand plot of Figure 7, while the impact damages [16] depend clearly on very high traffic conditions, on the contrary of expectations, track scaling [14] characterises tunnels with medium traffic conditions. This can be explained by the more frequent maintenance that characterises tunnels on axes with high traffic volumes and which obviously reduces the degradation rate. Traffic also causes the projection of water on the tunnel walls. This water together with de-icing salts particles contribute to gutter and side 
walls corrosion up to $1.5 \mathrm{~m}$ height [4], [5] and [6]. Nonetheless, it is interesting to observe that disorders caused by de-icing salts corrosion may also affect tunnels with low traffic. This can be ascribed to the fact that a significant number of low traffic tunnels are very old and/or are located at a high altitude. Actually, both quantity of de-icing salts (the applications frequency mainly depends on weather conditions) and concrete quality (which mainly depends on the age of the structure) contribute to lining deterioration by de-icing salts. Finally, the corrosive action of exhaust gases, together with humidity, can contribute to efflorescence formation [2] on the concrete of the lining.

\section{Fig. 7}

Other correspondence analysis results are shown in Figure 8.The graph on the left (Figure 8-1) shows the influence of tunnel age (i.e. service life duration calculated from the commissioning year) on the disorders development. Due to the weathering process of building materials, 40 years old structures as several Swiss National Roads tunnels may show serious disorders of the concrete lining, i.e. crumbling with local failure and fragments fall [13]. Also track conditions [14] depend on service life length. Furthermore, disorders due to ice formation [12] characterise the very old tunnels, probably because some were constructed without waterproofing system. This feature, together with the poorer quality of lining material and casting conditions may also explain fines transport in the drainage system [11]. On the other hand, as it is possible to observe, water incomes [1], efflorescence [2], calcium leaching [3], and cracks [8] cannot be associated to any particular age class.

As traffic grew regularly during the last 20 years, an analysis coupling the factors age and traffic was as well performed to estimate the traffic charge of each tunnel since its commissioning. This was achieved by integrating OFROU data on the average daily traffic volume between the 1985 and 2006. In addition to the disorders already identified as dependent on traffic, as impact damages [16] and de-icing salts aggression [5] and [6], in this case serious concrete degradations [13] have also been found connected to very high traffic charges. As a matter of facts, since traffic charges depend on traffic conditions during the whole tunnel service life and since old tunnels are characterised by low quality lining materials, serious disorders of the concrete lining [13] are observed for this category of tunnels.

Since the corrosive action of exhaust gases, together with humidity, contributes to concrete lining deterioration, the role of the ventilation system on the development of this kind of disorders has been investigated. As shown in Figure 8-2, tunnels with a natural ventilation (Na) or equipped with a longitudinal ventilation system (Lo) are affected by concrete deterioration, [3] and [13] and efflorescence 
[2]. Though in the case of natural ventilation the lining deterioration could be ascribed also to the age of the structures as most of tunnels without ventilation system are quite old, this explanation is not valid for tunnels equipped with a longitudinal ventilation system as this type of ventilation is also used in recent tunnels. Furthermore, it is interesting to observe that tunnels with a transversal (Tra) or semi-transversal (STra) ventilation system are apparently less affected by disorders caused by traffic pollution (assuming that the tunnel crown above the ventilation slab is as well inspected, as prescribed by the guidelines). Consequently, even in case of recent tunnels (i.e. good lining materials and casting quality) it can be stated that the concrete lining will less suffer of pathologies caused by aggressive atmosphere when the pollution is promptly evacuated from the tunnel.

\section{Fig. 8}

\subsubsection{Geological and hydrogeological conditions}

Together with operation and environment conditions, changes in the geological and hydrogeological conditions (e.g. weathering and creep of the rock mass, groundwater flow and chemical attack) may have an important influence on the tunnel degradation rate (Figure 9). Tunnels excavated in gypsum (evaporites) often shows calcareous concretions in the tunnel drainage system [17] together with efflorescence [2] and serious concrete lining deterioration [13]. Ice formations [12] develop more frequently in tunnels that cross discontinuous and blocky rock masses (e.g. carbonates and gneiss), while fines transport in the drainage system [11] characterises tunnels excavated in loose ground and marls and clayey media. The fact that also tunnels excavated in crystalline rocks show this type of disorder could be explained by the deterioration of sprayed concrete used as a temporary support. As it is possible to observe in Figure 9-1, invert heave [15] is mainly observed in formations characterised by swelling or squeezing potential such as anhydrites (evaporites), clay and marls and schists and phyllites; while water inflow [1] and crack and fissures [8] can be hardly associated to any precise geological class.

In addition to the significant dependency of the lining weathering on groundwater chemical composition and thus on rock mass formation (Wegmüller, 2001; Hobbs and Taylor, 2000), the type and position of the waterproofing system are as well determining (Figure 9-2). In particular, the absence of waterproofing causes the development of all those disorders that depend on groundwater flow into the tunnel: efflorescence [2], concrete lining weathering [3] and [13], and ice formation [12]. Calcareous concretions in the drainage system [17] seem also linked to the absence of waterproofing. Indeed, the groundwater can leak into the concrete lining and charge in Calcium which on the long term, can 
precipitate in the drainage system. This process which may take several years is supported by the fact that tunnels without waterproofing system are very old ones. Nonetheless, it is worthwhile to observe that this disorder is observed also in tunnels with a partial waterproofing system placed between the temporary and the final lining, probably due to the groundwater leaching through the shotcrete support behind the waterproofing membrane.

Disorders caused by the corrosive action of de-icing salts ([4], [5], and [6]) mainly affect tunnels with an internal waterproofing system. Usually placed only at the tunnel crown intrados, this system works as an umbrella. After percolating through the lining, the water moves along the extrados of the panels, and then it reaches and saturates the lower part of the side walls where these disorders are observed. Though invert heave up [15] seems to depend on waterproofing partial system, this should be considered as a bias of the correspondence analysis, because this disorder mainly occurs in tunnels with horseshoe shaped section generally equipped with a partial waterproofing system. Finally, since water incomes [1] affect the majority of tunnels it results quite difficult to associate this disorder to a specific waterproofing system.

The influence of tunnel depth on the development of disorders is shown in Figure 9-3. It has been possible to observe that tunnel invert deformation [15] is mainly related to deep tunnels, so it is for track scaling [14] which is sometimes a manifest symptom of invert heave. For other disorders such as water infiltration [1] or cracks and fissures [8], it is interesting to see that there is no clear evidence of depth influence on their development. This is due mainly to the fact that overburden can be considered as an additional influence factor that should be coupled with geological and hydrogeological conditions. Nonetheless, in many shallow tunnels and in zones near portals (i.e. overburden less than one or two times the tunnel diameter), cracks and fissures are frequently observed, probably due to the bending of the lining caused by the asymmetric loading. Also, water ingress and other disorders related to water infiltration are observed, most probably because groundwater and surface water find an easy way to the tunnel through weathered rocks or loose ground masses.

\section{Fig. 9}

\subsection{Main conditions influencing tunnel degradation}

This last step in the data analysis process is necessary in order to confirm the preliminary findings of the correspondence analysis about the main influence factors and to identify for each disorder the respective conditions within all influence factors modalities. 
Table 4 summarises, for each disorder, the main influence factors determined according to the analysis performed in Section 3.2. Five dependencies are marked by a question mark ('?’) because they are highlighted in the specific literature (e.g. CETu, 2004, Fed. Highway Admin. and Fed. Transit Admin., 2003b), but no evidence has been found in the correspondence analysis results.

Table 4

Among the several attributes/modalities of each influence factor, only some of them contribute to the development of identified disorders. To highlight the relationship between factor modalities and observed disorders, further analyses have been performed. For each influence pointed out in Table 4 between a factor and an observed disorder, the analysis was performed as follows:

1. Selection of the tunnels affected by the disorder according to the last principal inspection observations;

2. Distribution of the tunnels population into modality classes of the corresponding influence factor (see Section 2.2);

3. Evaluation of the probability that a tunnel belonging to a certain modality class shows actually the disorder. For each factor modality class, the conditional probability of disorder occurrence is calculated as follows:

$$
P(\text { disorder factor modality })=\left(\frac{\text { number of affected tunnels }}{\text { total number of tunnels }}\right)_{\text {factor modality class }}
$$

Table 5 summarises the results of this last step of the analysis process. A significant influence corresponds to a conditional probability higher than $45 \%$; a medium influence to values between $45 \%$ and $30 \%$ and a slight influence stands for values between $30 \%$ and $15 \%$. No influence is considered for a conditional probability lower than $15 \%$.

Table 5

Also the joint probability describing the combination of influence factors modalities has been evaluated. The probability of two events in conjunction, if the two factors are independent, corresponds to the product of their individual probabilities. The interaction has been calculated by considering two factors at once, taking into account only the modalities characterised by the highest values. Moreover, in order to avoid the problem of analysing a high number of few populated classes of tunnels, losing in terms of results relevance, the conditional probability expressing the interaction between dependent factors has not been calculated. 
Since the selected influence factors are current tunnel features, Table 5 allows determining for each disorder, symptom of major tunnels pathologies, the modalities that mainly contribute to its occurrence and the most favourable scenario for its development.

[1] Though the probability of observing water incomes and moisture is quite high in the National Road tunnels (i.e. 56 affected tunnels over 122 inspected) these disorders are influenced by waterproofing system, geological and hydrogeological conditions and tunnel depth. Higher probability values characterise tunnels without sealing (No) and tunnels only equipped with an internal waterproofing system (I). Also, geological conditions influence the probability of observing water incomes in the tunnel. In particular, this value is higher in discontinuous rock masses (Cry and Ca). Keeping in mind that the depth classification of the tunnels is based on their maximum overburden, the influence of this factor is on a slant. Actually, water incomes are mainly observed near the portals and in the shallow zones of the tunnels. The interaction of the factors waterproofing system, geological and hydrogeological conditions and tunnel depth has a strong influence too on the development of this disorder, e.g. the absence of waterproofing system in tunnels excavated in fissured rock masses are joint conditions that aggravate water incomes. Furthermore, when the tunnel is at a low depth and the geology allows an easy way for the water to reach the tunnel without an appropriate waterproofing system, water inflow may be observed more frequently, especially during and after rain falls.

[2] Efflorescence mainly depends on groundwater action. High probability values characterise tunnels without waterproofing system as well as tunnels that cross gypsum (evaporites, i.e. high sulphates content) and acid gneissic formations. The possibility of aggressive groundwater leaking inside a tunnel without waterproofing system is frequently documented in literature as a major scenario for efflorescence development (Romer et al., 2003; Planel et al., 2006). The aggressive atmosphere inside the tunnel due to traffic pollution can be considered only as a secondary influence factor as well as longitudinal and natural ventilation systems. As efflorescence mainly affects tunnel crown, there is a potential bias in the results for the transversal (Tra) and semi-transversal (S-Tra) ventilation systems as one might wonder whether and how the lining conditions above the ventilation slab have been investigated during the principal inspections. So it is for tunnel equipped with an internal waterproofing system (I) because the panels prevent the disorder identification at the tunnel crown.

[3] Weathered surfaces due to calcium leaching, staining and calcareous concretions mainly depend on hydrogeological conditions. Water circulating in evaporites and crystalline rocks seems to be the 
principal cause of this disorder affecting concrete linings. Obviously, since this disorder is strongly influenced by the presence of water, other important factors are shallow depth and absence of waterproofing system allowing water circulation around and inside the cavity. Though only as a secondary factor, also ventilation system seems to play a role in the development of this disorder which characterises only tunnels with natural or longitudinal ventilation systems. Nonetheless, as already explained, the presence of a ventilation slab characterising transverse and semi-transverse ventilation systems may hide this kind of disorder.

[4] Concrete spalling and delaminated concrete, together with plain concrete damage by de-icing salts (disorder no. [5]) and corrosion of steel bars by Chlorides for reinforced concrete linings (i.e. disorder no. [6]) depend on traffic conditions. Overall, their probability increases with increasing daily traffic volume. However, a high probability of reinforced concrete corrosion [6] is also found in case of low traffic volumes. For example, this happens for tunnels on roads located at a high altitude and thus subjected to harsh weather conditions with severe temperature decrease that require high quantities of salt for low traffic volumes. Moreover, in those tunnels the maintenance frequency is probably lower than in tunnels with high daily traffic volume. A particular remark should be done about the influence of the waterproofing system. The disorders [4], [5] and [6] depend indeed on the concrete lining saturation which may increase in case of internal waterproofing system. The possibility of water to saturate the lower part of the tunnel side walls together with the de-icing salts projection by rolling traffic are particularly aggressive for concrete lining (reinforced or not).

[5] see disorder [4]

[6] see disorder [4]. Though few tunnels have a final lining made of reinforced concrete, the corrosion of steel bars is a widespread disorder observed in almost half of the tunnels population for which the information about last principal inspection is available (see Table 3). This is due to the fact that it affects mainly the lower part of the tunnel side walls which is made of reinforced concrete (not for supporting purposes but mainly for structural connections). It results from the saturation of the concrete and from the projection of de-icing salts particles by rolling traffic. This disorder is observed in the 4 tunnels with an internal waterproofing system (I) and in many tunnels with a partial waterproofing placed between the support and the lining $(\mathrm{Pm})$ or at the support extrados (Pe).

[7] This disorder has not been recorded during last tunnels principal inspections (see Table 3). 
[8] Cracks and fissures are more frequent in shallow tunnels, possibly due to the loss of axisymmetric loading conditions. However as several causes may contribute to fissures development, a detailed characterisation of type, aspect, position, length and width would be required (and collected in the data base) to allow identifying their origin.

[9] As mentioned previously, this disorder, observed only once during last principal inspections, has not been considered significant for this analysis.

[10]This type of disorder has not been recorded during last tunnels principal inspections (see Table 3).

[11]Fines transport in the drainage system depends on tunnel geological conditions: this disorder mainly occurs in tunnels excavated in loose ground masses and clay formations. Also, the combination of lower quality concrete, poor casting conditions and absence of waterproofing system which are typical of old structures (A) is favourable to its development.

[12]As expected, ice formation affects mainly tunnels without waterproofing system. Since this feature is mainly characteristic of tunnels constructed before the seventies, this explains also the high probability value found for very old tunnels (A). On the contrary this disorder is not observed in tunnels with a total waterproofing. The hydrogeological conditions influence also the development of this disorder: higher probability values characterise indeed tunnels excavated in fissured and blocky rock masses where groundwater may find an easier way to the tunnel.

[13]Due to the low number of observations (i.e. 6 times during the last principal inspections) lining degradation till crumbling has been considered as the final stage in the life of tunnel concrete lining. Indeed it affects very old tunnels often characterised by a lower quality of construction materials and without waterproofing system. The importance of the age of the tunnel results also from the dependency of this disorder from the total traffic charge during tunnel service life (Section 3.2.1). In addition to the probability values reported in Table 5 for the daily average traffic volume, the probability values were also calculated by considering the total traffic charge: Low $=0 \%$; Medium = $0 \%$, High $=0 \%$, Very High $=22 \%$. These results clearly show that very high traffic charges, characteristics among others of a long duration service life, may influence the development of this disorder. As for the geological conditions, evaporites are found to cause problems due to the swelling pressure that this kind of rock mass may develop with time in case of chemical reaction with water (i.e. anhydrite) and to the aggressive action of groundwater when charged in sulphates (gypsum) which 
may cause lining weathering till crumbling. Such lining instabilities affect as well tunnels excavated in blocky rock masses due to unexpected local loads to bear.

[14]Track scaling has a high probability to affect old tunnels, because track damage increases with time. Against any expectations, the traffic seems not to influence the development of this disorder. This can be explained by the more frequent track maintenance that characterises tunnels with very high traffic conditions. It point out the importance of conservation procedures for reducing degradation rate. As shown in Figure 9-3, high overburden seems to influence as well the development of track scaling which can be considered also a manifest symptom of tunnel invert heave up [15].

[15]Being a typical symptom of rock mass delayed behaviour, the probability of observing invert heave up is higher in deep tunnels, due to the high confinement conditions. Also geological conditions are important since the higher probability values have been found for tunnels excavated in marls and clayey media and evaporites often showing swelling and squeezing behaviours.

[16]As it is commonly observed, impact damages are mainly the consequence of very high traffic volumes.

[17]Drainage system obstructions by calcareous concretions mainly affect tunnels without waterproofing system (No). Indeed, groundwater may increase its Calcium content when in contact with the poor quality concrete lining of very old tunnels. Also when a partial waterproofing system is placed between the support and the definitive lining (Pm), the percolating groundwater may increase its Calcium content before reaching the drainage system when leaching through the shotcrete support. The drainage system conditions depend as well on the geological and hydrogeological conditions. This disorder mainly affects tunnels excavated in evaporites and carbonates. The high probability value that characterises marls and clayey media can be explained by the low permeability of this kind of rock mass. Slowly percolating around the cavity, the groundwater may indeed charge in Calcium content from the temporary shotcrete support.

\section{RESULTS DISCUSSION}

Tunnel degradation diagnosis means assessing the actual tunnel conditions, determining the main causes of the observed disorders, and developing an appropriate strategy for maintaining the structure, ensuring its safety and serviceability. Appropriate conservation techniques will result from a good identification and understanding of the degradation mechanisms, including their speed. Data collection and analysis, 
since tunnel construction and during its service life, may help the tunnel owner in following the structure evolution. Indeed, by taking into consideration all tunnel features such as geometry, geological and hydrogeological conditions, age, construction techniques, operational conditions and material quality together with inspections results, tunnel long term serviceability and behaviour assessment may be improved.

\subsection{Tunnel Data Base}

The analysis performed in Section 3 is based on the information collected in the Tunnel Data Base (Section 2). Most of the data were collected directly by consulting cantonal archives for road infrastructures. Some were provided by cantonal administrations and others were taken from specific literature and/or G.I.S. maps. This variety of sources and people undoubtedly influences the quality and homogeneity of the compiled information. However, the data consistency was maximised by identifying and coding modalities for each considered factor.

During inspections, the perception of tunnel global conditions is mainly based on lining quality and aspect, which could give the impression that tunnel disorders are only caused by lining pathologies.

Actually, geological and hydrogeological conditions have a major role in the development of several disorders and should consequently be documented. In the compiled data base, $30 \%$ of the tunnel population is documented with a geological profile (but only the $15 \%$ were provided directly by cantonal administrations) and while 55\% is only characterised by a list of crossed lithologies / geological units. For the remaining portion (15\%), no geological data was even available! To supply the lack of data and to homogenise the geological and hydrogeological information, G.I.S. documents were used to roughly describe the probable geology at each tunnel location. As the precision and scale of G.I.S. maps are not comparable at all to those of data compiled during tunnels construction, the considered geological and hydrogeological data have a rather poor quality and allow only general considerations about pathologies development. Major improvements in the data analysis and in the identification of degradation processes could be achieved if an accurate documentation of the geological and hydrogeological conditions was available at the tunnel scale. Moreover, construction details such as information about temporary support, definitive lining, advance rate, accidents during construction could help identifying and localising some pathologies. At present, only few guidelines for road infrastructures maintenance (CETu, 1998; CETu, 2005) specify the importance of collecting those data. 
Together with a detailed characterisation of the tunnel conditions and features at the local scale, inspection results and disorders observations are necessary for correctly interpreting the tunnel degradation phenomena. According to the guidelines from the Swiss National Roads Authority (OFROU, 1998/2005), principal inspections should be documented by appropriate reports. Nonetheless, the main difficulties encountered during data collection result from the lack of a standardised way of recording inspection observations, in particular for what concerns disorders location and features (e.g. extent, severity). The actual system can be improved by documenting disorders observations in a more detailed way, showing their position and severity, e.g. by means of sketches, (as it is already done in France by CETu and in Switzerland by the National railway company and Ticino Canton, for example).

\subsection{Data Analysis}

As the data base was created and compiled for the Swiss National Roads tunnels, the data analysis presented in Section 3 and the statements made in this framework are valid for road tunnels with a concrete lining. Moreover, the study is limited to excavated tunnels and does not consider cut and cover tunnels. As a matter of facts, due to their different shape and construction method, those structures interact differently with the surrounding ground and may consequently show other pathologies and disorders. Finally, because Swiss tunnel inspectors just begin recently to be aware of the Alkali-Aggregate Reaction (AAR) in concrete structures, disorders related to this deterioration process are not considered in the present analysis.

The main purpose of the presented analysis is to identify the influence factors involved in degradations processes that affect roads tunnels during their service life. The contribution of each tunnel feature to disorders development has been analysed by means of multivariate statistics methods (i.e. correspondence analysis) which allow evaluating the disorder dependency on influence factors if a sufficient number of observations has been recorded. Since for some of the disorders only a few numbers of cases have been observed and recorded, it has not been possible to select all the influence factors that contribute to their development. On the other hand, although these data analysis methods are powerful tools, the rather poor quality of some data available in the TDB limits somewhat the conclusions that can be drawn.

The data analysis is mainly based on the mean number of disorders per tunnel. Though this is a quite important simplification to find correspondences between tunnel features (including geological and hydrogeological conditions) and disorders type, calculating the mean number of disorders per meter of 
tunnel (i.e. dividing the total number of disorders by the length of the tunnel) instead of per tunnel, has been as well considered, but not adopted as it could result in misjudgements (e.g. if the disorders are concentrated in a certain portion of the tunnel). A finer analysis would require indeed a detailed description (i.e. meter by meter) of tunnel local conditions and features (i.e. construction and operation conditions together with disorders observation and location). Actually, as discussed in the previous section, for the majority of tunnels in the data base, neither the geological and construction data nor the disorders observation during the principal inspections are at present sufficiently detailed to refine the analysis.

In the data analysis, only disorders data coming from the last principal inspection have been taken into account. For moving towards a degradation diagnosis and a maintenance planning, it is necessary to integrate successive inspections results and to predict the tunnel behaviour with time by means of degradation models (Sandrone and Labiouse, 2010). The importance of updating the collected data is prescribed by guidelines of several countries (Dir. Générale des Transports Intérieurs and Min. des Transports, 1980; Highways Agency et al., 2003; Fed. Highway Admin. and Fed. Transit Admin., 2003a). In the future, a regular update of the data base with observations from successive inspections would be useful to follow the evolution of identified disorders and to estimate the tunnel degradation rate.

\subsection{Tunnel conditions evaluation (“Global vs. Disorder Index”)}

For developing a conservation strategy plan, a life-cycle cost analysis can be made by comparing maintenance options in both short and long term rehabilitations, which often consist in a decision of increasing the maintenance frequency or planning extensive repairs. Indeed, according to (Yamazaki and Tsuburaya, 2001), the maintenance policy may change the total amount of money necessary for guaranteeing an acceptable service and safety quality level of the tunnel. The analysis should integrate information about tunnel conditions together with a socio-economical evaluation mainly based on the importance/location of the tunnel on the whole road network. Though this topic is not treated in this framework, some considerations can be done in the perspective of indexing actual tunnel conditions. Following the procedure presented in this paper for identifying the main factors that influence tunnel degradation, and considering the obtained results (Section 3.3), the global index commonly used by tunnel inspectors, in spite of its simplicity, can not be considered as an exhaustive index for describing tunnel conditions. An interesting and alternative approach could be the use of several indexes describing tunnel conditions with respect to the identified disorders. Pathology location and severity (i.e. affected 
zone extension) could be used as an internal weight for each index. Moreover, the identification of main scenarios that determine degradation rate could help in focusing attention (and means) on major degradation processes. Finally, after a good diagnostic of the tunnel conditions, social and economical factors will determine the tunnel owner decision. This procedure can be resumed in three main steps:

1. tunnel initial condition evaluation to assess the degradation potential of each structure, considering the identified pathologies,

2. estimation of degradation rate based on identified pathology scenarios,

3. socio-economical evaluation of the structure (CETu, 2005; Brühwiler, 2004) and cost benefit analysis.

\section{SUMMARY AND CONCLUSION}

Tunnel degradation can be identified by several disorders. Within the range of problems observed during tunnel inspections some of the disorders slightly affect the tunnel lining, and thus only decrease the tunnel's serviceability level, while others can be symptomatic of strength deterioration, and then reduce the tunnel's structural performance and safety. The diagnosis of tunnel conditions needs to go through several steps:

I. description of the disorders and their extent,

II. identification of their causes and of the main factors that contribute to their development,

III. assessment of their effects on the tunnel stability and evolution in the long term.

As suggested by the guidelines of Road Authorities of several countries (e.g. OFROU, 1998/2005; CETu, 1998; Highways Agency et al., 2003; Fed. Highway Admin. and Fed. Transit Admin., 2003a), a good diagnosis is based on the analysis of well structured and updated data collections. As tunnel conditions result from its degradation potential, due to initial conditions (e.g. geology, construction features), and its degradation rate, due to service life and operation conditions, data bases should regroup information on construction, geology, environment, operation and maintenance (including inspection reports).

After a brief portrait of main features characterising Swiss National Roads tunnels, this paper describes a specific data analysis process for investigating tunnel degradation (Figure 1 and Figure 5). Exploratory data analysis techniques have been used for improving disorders identification and the diagnosis of tunnel conditions that follows principal inspections and which is a part of the tunnel conservation process. The whole analysis procedure can be summarised as follows: 
1. Initial conditions - Degradation potential. The tunnel degradation potential has been analysed by considering tunnel initial conditions such as construction features (e.g. commissioning year, construction method and waterproofing system) as well as geological and hydrogeological conditions. These features being potential influence factors, their effects on the degradation potential have been evaluated by considering the mean number of disorders observed in the tunnels during their last principal inspection per each factor modality.

2. Service life and tunnel operation - Degradation rate. With respect to the environment and the operation conditions (e.g. traffic volume, ventilation system, de-icing salts use) the factors that influence disorders development have been identified. By means of Correspondence Analyses it has been possible to determine systematic dependencies between disorders and influence factors.

3. Degradation scenarios. After having identified the main influence factors from the two previous analysis steps, representative scenarios of disorder development have been finally described. For any selected influence factor, the probability of occurrence of the related disorders has been evaluated for each factor modality or combination of factor modalities.

The analysis shows that lining disorders identified during tunnel inspections may be caused by rock mass degradation and delayed behaviour, tunnel features, environment and operation conditions. For example, it is observed that:

- Geological and hydrogeological conditions are of prime importance in tunnel degradation. Tunnel disorders such as cracks and fissures, deformations and invert heave, are correlated with delayed behaviour and weathering potential of the rock mass. A significant number of disorders are observed in schists and phyllites, and in marl and clayey media. Other problems affecting the drainage system such as calcareous concretions and fines transportation are as well related to the rock mass and water circulation around the tunnel.

- The influence of groundwater on the tunnel stability depends on both its chemical composition and circulation around the tunnel. Chemical reactions between water and concrete reduce the concrete resistance and as a consequence the tunnel stability on the long term, moreover, the redistribution of pore water pressures inside the rock mass and on the lining may induce significant delayed effects and a progressive change in stability conditions. The degradation potential of both mechanisms depends on the type of rock formation (porous, discontinuous, karstic) and waterproofing system. 
- Construction features such as commissioning year, construction method, waterproofing system, quality of building materials have a relevant influence on the tunnel degradation potential and development of future disorders. For example, the absence of waterproofing system as well as the poorer quality of lining material and casting conditions that often characterise old tunnels facilitate the interaction of water and lining accelerating the rate of the weathering processes.

- Since the corrosive action of exhaust gases, together with humidity, contributes to concrete lining deterioration, the environment and operation conditions in the tunnel influence the lining degradation rate. Concrete pathologies caused by the aggressive atmosphere inside the tunnel have indeed been found related to the traffic charge and the way the pollution is removed (i.e. type of ventilation system). Traffic also causes the projection of water on gutter and side walls, which together with the use of de-icing salts during winter season induce corrosion, scaling and deterioration of the concrete.

Although the obtained results confirm common expectations, this data analysis of Swiss road tunnels revealed quite useful for improving the understanding of tunnel degradation mechanisms and pointing out the factors that mainly contribute to their development. Once the degradation potential and rate are known according to the tunnel initial and operation conditions, a better description of evolution scenarios can be expected, which may help in making decisions on preventive maintenance (i.e. type of intervention and frequency). The results can be also used for prioritising interventions (i.e. by localising tunnels characterised by critical conditions with respect to the development of a selected pathology). Finally, the results may also improve the design of new structures by taking into consideration their actual conditions.

\section{ACKNOWLEDGEMENTS}

The authors thank the Swiss Federal Road Authority OFROU for its financial support (grant no. FGU2003/002). They are also grateful to the Maintenance and Inspection team from CETu (France) for useful suggestions during the conception of the data base, and J.-F. Mathier for his precious help during data collection.

\section{REFERENCES}

AFTES Association Française des Travaux en Souterrain, WG no. 14, 1999. Text of recommendations on Diagnosis Methods for Lined Tunnels. Tunnels et ouvrages souterrains, 86-105. [FR4]

Asakura, T., Kojima, Y., 2003. Tunnel maintenance in Japan. Tunn. Undergr. Sp. Tech.,18, 2-3, 161-169. $[\mathrm{J}]$

Bouvard-Lecoanet, A., Colombet, G., Esteulle, F., 1988. Chapitre 11: Entretien et réparation des tunnels. in Ouvrages souterrains. Conception, réalisation, entretien. Presses de l'Ecole Nationale de Ponts et de Chausées, Paris, pp. 247-253. [FR2] 
Brühwiler, E., 2004. Maintenance des ouvrages: notes du cours EPFL. Ecole Polytechnique Fédérale de Lausanne.

Carranza-Torres, C., Zhao, J., 2009. Analytical and numerical study of the effect of water pressure on the mechanical response of cylindrical lined tunnels in elastic and elasto-plastic porous media. Int. J. Rock Mech. Min., 46, 3, 531-547.

CETu, Min. Equipement des Transports et du Logement and Direction des Routes, 1998. Guide pour la surveillance, l'entretien et la conservation des tunnels routiers. France, p. 127. [FR3]

CETu, Min. Equipement des Transports et du Logement and Direction des Routes, 2004. Guide de l'inspection du génie civil des tunnels routiers. Des désordres vers le diagnostic. France, p. 80. [FR5]

CETu, 2005. Personal communication.

Dir. Générale des Transports Intérieurs and Min. des Transports, 1980. Instruction technique pour la surveillance et l'entretien des ouvrages d'art. Deuxième partie. Fascicule 40, Tunnels - Tranchées couvertes - Galeries de Protections. DRCR Direction des Routes et de la Circulation Routière, Paris, p. 47. [FR1]

Favre, R., Andrey, D., Suter, R., 1987. Maintenance des ouvrages d'art. Méthodologie de surveillance. Lausanne, p. 306. [CH1]

Fed. Highway Admin., Fed. Transit Admin., 2003a. Highway and Rail Transit Tunnel Inspection Manual. USA, p. 103. [US1]

Fed. Highway Admin., Fed. Transit Admin., 2003b. Highway and Rail Transit Tunnel Maintenance and Rehabilitation Manual. USA, p. 103. [US2]

Filipponi, M., 2009. Spatial analysis of karst conduit networks and determination of parameters controlling the speleogenesis along preferential lithostratigraphic horizons. Thèse EPFL no. 4376. $f$ cole Polytechnique Fédérale de Lausanne, p. 305.

Gärber, R., 2003. Design of Deep Galleries in low Permeable Saturated Porous Media. Thèse EPFL no. 2721. fcole Polytechnique Fédérale de Lausanne, p. 224.

Greenacre, M.J., 1984. Theory and applications of Correspondence Analysis. Academic Press, London, p. 364.

Grobbelaar, C., 1994. The Degradation and Failure of Concrete Linings around Water Conveyance Tunnels. Tunn. Undergr. Sp. Tech., 9, 1, 67-71.

Highways Agency, Scottish Executive Development Dep., Welsh Assembly Gov. Llywodraeth Cynulliad Cymru, Dep. for Regional Development Northern Ireland, 2003. Maintenance of Road Tunnels in Design Manual for Road and Bridges, United Kingdom, p. 39. [UK]

Hobbs, D.W., Taylor, M.G., 2000. Nature of the thaumasite sulphate attack mechanism in field concrete Cement Concrete Res., 30, 4, 529-533.

Iftimie, T., 2001. Prolongation de la durée de vie des tunnels anciens par des travaux de réhabilitation. Proceedings, Progress in Tunnel after 2000. AITES-ITA World Tunnel Congress, Milano, Vol. 2, pp. 603-610.

Infravia, 2000. Gallerie: adeguamento, manutenzione e arredo delle opere in sotterraneo esistenti. Convegni Infravia, SIG Società Italiana Gallerie, Verona, Italy. [I1]

Locatelli, L., Di Marco,G., Zanichelli, C., Jarre, P., 2001. Rehabilitation of Highway Tunnels Techniques and Procedures. Proceedings, Progress in Tunnel after 2000. AITES-ITA World Tunnel Congress, Milano, Vol. 2, pp. 653-664. [I2]

Merz, C., Hammerschlag, J.-G., 2000. Réactions alcali-granulats (1ère et 2ème parties). Bullettin du ciment du TFB, Service de recherches et conseils techniques de l'industrie suisse du ciment, Wildegg, nos. 5, 9.

Moreau, J., Doudin, P.-A., Cazes, P., 2000. L'Analyse des correspondances et les techniques connexes. Approches nouvelles pour l'analyse statistique des données, 32, Springer, Berlin, p. 265. 
Nishisato, S., 2004. Dual Scaling. Section Scaling: Chapter1. The SAGE Handbook of Quantitative Methodology for the Social Sciences, D. W. Kaplan, Sage Publications Inc., London, p. 24/528.

Office Fédéral des Questions Conjoncturelles, 1993. Le diagnostic des ouvrages de génie civil. Manuel pour ingÉnieurs civils. Berne, p.180. [CH2]

Office Fédéral des Questions Conjoncturelles, 1994. Maintenance des ouvrages en tunnel. Berne, p. 113. [CH3]

OFROU Office fédéral des routes, 1998/2005. Surveillance et entretien des ouvrages d'art des routes nationales. Berne, p. 33/48. [CH5]

OFROU Office fédéral des routes, 2002. Prise en considération de l'entretien dans l'Élaboration des projets lors de la construction des routes nationales. Berne, p. 39. [CH6]

OFROU Office fédéral des routes, 2004. Directive pour la saisie des donnÉes des ouvrages d'art des routes nationales dans KUBA. Berne, p. 89.

Parker, H.W., Robinson, R.A., Godlewski, P.M., Hultman, W.A., Guardia, R.J., 2001. Tunnel Rehabilitation in North America. Proceedings, Progress in Tunnel after 2000. AITES-ITA World Tunnel Congress, Milano, Vol. 2, pp. 697-704. [US3]

Planel, D., Sercombe, J., Le Bescop, P., Adenot, F., Torrenti, J.-M., 2006. Long-term performance of cement paste during combined calcium leaching-sulfate attack: kinetics and size effect. Cement and Concrete Res., 36, 137-143.

Richards, J.A., 1998. Inspection, maintenance and repair of tunnels: International lessons and practice. Tunn. Undergr. Sp. Tech., 13, 4, 369-375. [SA]

Romer, M., Holzer, L., Pfiffner, M., 2003. Swiss tunnel structures: concrete damage by formation of thaumasite. Cement Concrete Comp., 25, 1111-1117.

Sandrone, F., Labiouse, V., 2010. Analysis of the evolution of road tunnels equilibrium conditions with a Convergence-Confinement approach. Rock Mech. Rock Eng., 43(2), 201-218.

Sandrone, F., Labiouse, V., Mathier, J.-F., 2007a. Data Collection for Swiss Road Tunnels Maintenance. Felsbau, 1, 8-14.

Sandrone, F., Labiouse, V., Mathier, J.-F., 2007b. Preliminary Identification of Swiss Road Tunnels Pathologies based on Geotechnical G.I.S. data. Proceedings, 11th ISRM Congress - The Second Half Century of Rock Mechanics, Lisbon, Vol. 2, pp. 999-1002.

SIA Société suisse des ingénieurs et des architectes, 1997. SIA 469 Conservation des ouvrages, Zurich, p. 24. [CH4a]

SIA Société suisse des ingénieurs et des architectes, 2003. SIA 262 Construction en béton, Zurich, p. 94. [CH4b].

SIA Société suisse des ingénieurs et des architectes, 2003. SIA 260 Bases pour l'élaboration des projets des structures porteuses, Zurich, p. 44. [CH4c]

Széchy, K., 1966. The Art of Tunnelling. Akadémiai Kiadó, Budapest, p. 891.

VSS, 2000. SN 640908 Gestion de l'entretien; évaluation de tronçons de route dans le réseau - évaluation fonctionnelle, Zurich, p. 12.

Wegmüller, M.C., 2001. Einflüsse des Bergwassers auf Tiefbau/Tunnelbau. Stäubli AG, Zurich, p. 215.

Worthington, S.R.H., 2003. A comprehensive strategy for understanding flow in carbonate aquifers. Speleogenesis and Evolution of Karst Aquifers 1, 1, www.speleogenesis.info: 8, re-published from: Palmer, A.N., Palmer, M.V., Sasowsky, I.D. eds., 1999. Karst Modeling: Special Publication 5, The Karst Waters Institute, Charles Town, West Virginia, pp. 30-37.

Yamazaki, T., Tsuburaya, Y., 2001. A case study on the period for the economically optimal repairs of RC tunnels suffering of chloride damage. Proceedings, Progress in Tunnel after 2000. AITES-ITA World Tunnel Congress, Milano, Vol. 2, pp. 777-784. 


\subsection{List of codes used in the text (Table 1)}

[CH1]: Favre et al. (1987)

[CH2] Office Fédéral des Questions Conjoncturelles (1993)

[CH3] Office Fédéral des Questions Conjoncturelles (1994)

[CH4a] SIA 469 (1997)

[CH4b] SIA 262 (2003)

[CH4c] SIA 260 (2003)

[CH5] OFROU (1998/2005)

[CH6] OFROU (2002)

[FR1] Dir. Générale des Transports Intérieurs and Min. des Transports (1980)

[FR2] Bouvard-Lecoanet et al. (1988)

[FR3] CETu (1998)

[FR4] AFTES (1999)

[FR5] CETu (2004)

[I1] Infravia (2000)

[I2] Locatelli et al. (2001)

[UK] Highways Agency et al. (2003)

[US1] Fed. Highway Admin. and Fed. Transit Admin. (2003a)

[US2] Fed. Highway Admin. and Fed. Transit Admin. (2003b)

[US3] Parker et al. (2001)

[J] Asakura and Kojima (2003)

[SA] Richards (1998)

\subsection{Data sources}

OFEV Office fédéral de l'environnement - Federal Office for the Environment: http://www.hydrodaten.admin.ch

OFROU Office fédéral des routes - Federal Roads Office: http://www.astra.admin.ch

Swisstopo Office Fédéral de la Topographie, 2000. Atlas hydrologique de la Suisse. Berne, Switzerland 


\section{List of Figures:}

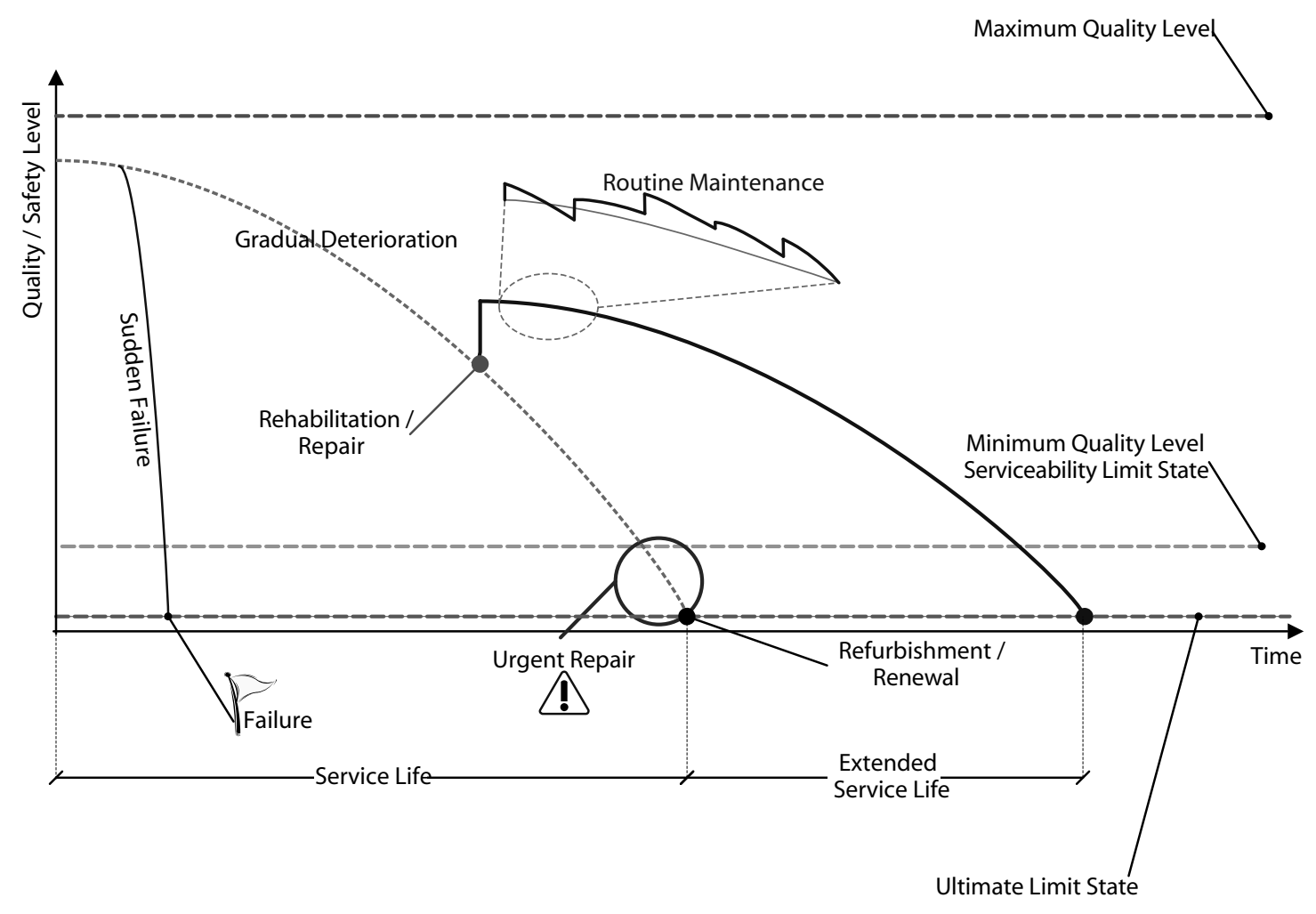

FIGURE 1. Time evolution of the quality level of a tunnel and effect of conservation practices during service life.

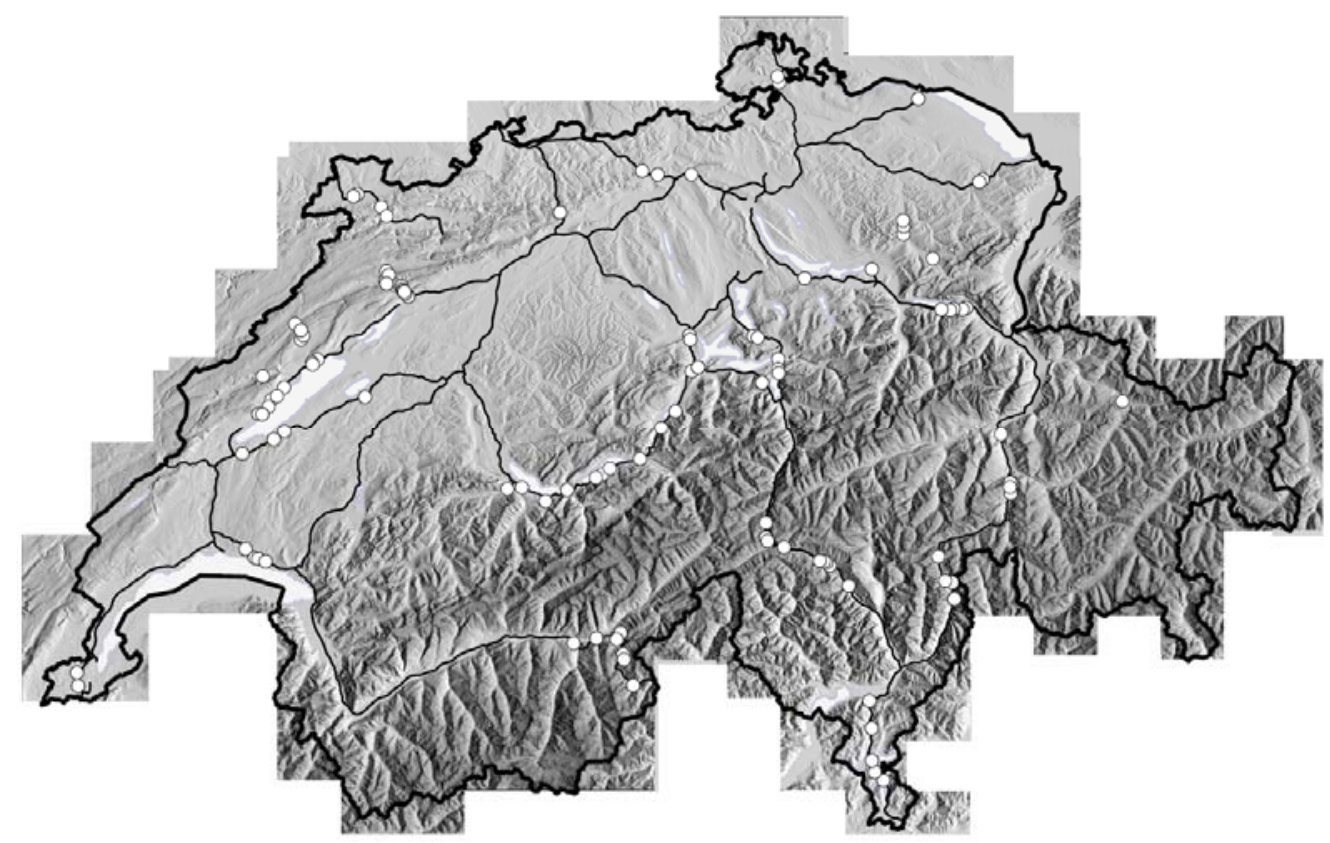

FIGURE 2. G.I.S. representation of all the tunnels of the Swiss National Roads network that are documented in the data base. Each tunnel is georeferenced by means of the $(X, Y)$ coordinates of its centre point. Though third class National Roads are not represented in this map, their tunnels have been also included in the data base. 

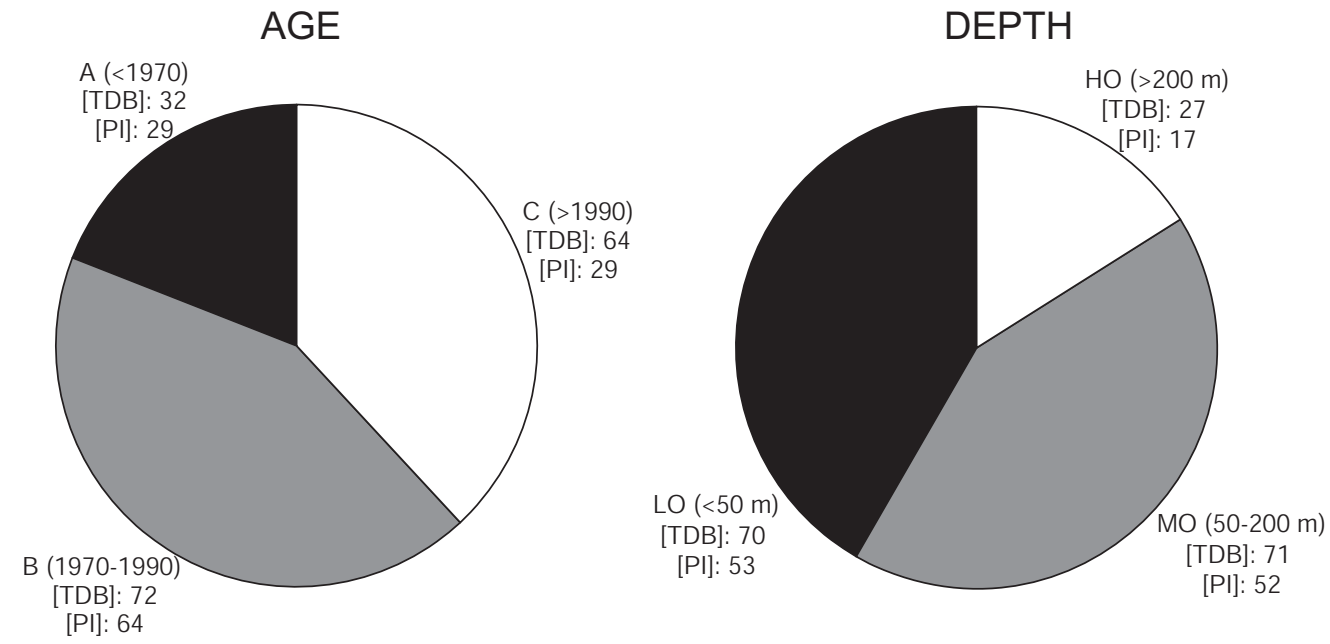

FIGURE 3. Distribution of Swiss National Roads tunnels population according to their age, on the left, and depth, on the right. In the pie chart it is specified: [TDB] the number of tunnels recorded in the TDB and [PI] the number of inspected tunnels (i.e. for which there is information about the last principal inspection). 

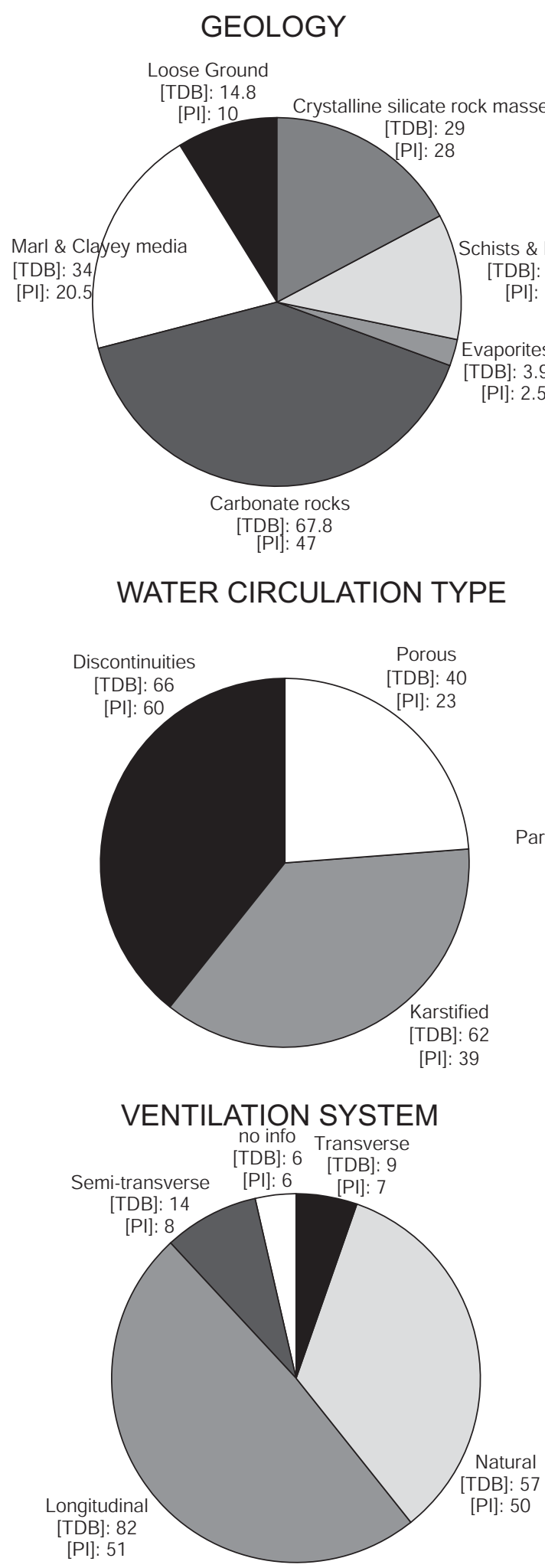

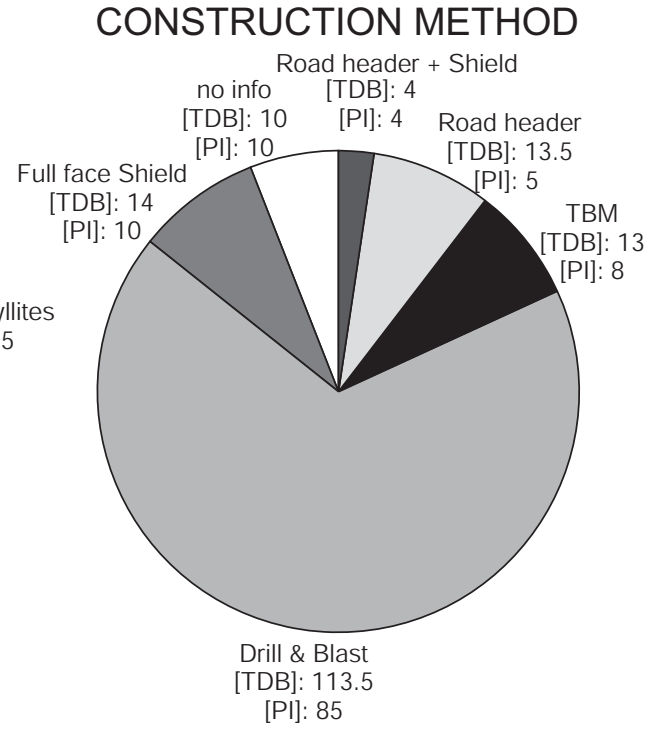

WATERPROOFING SYSTEM
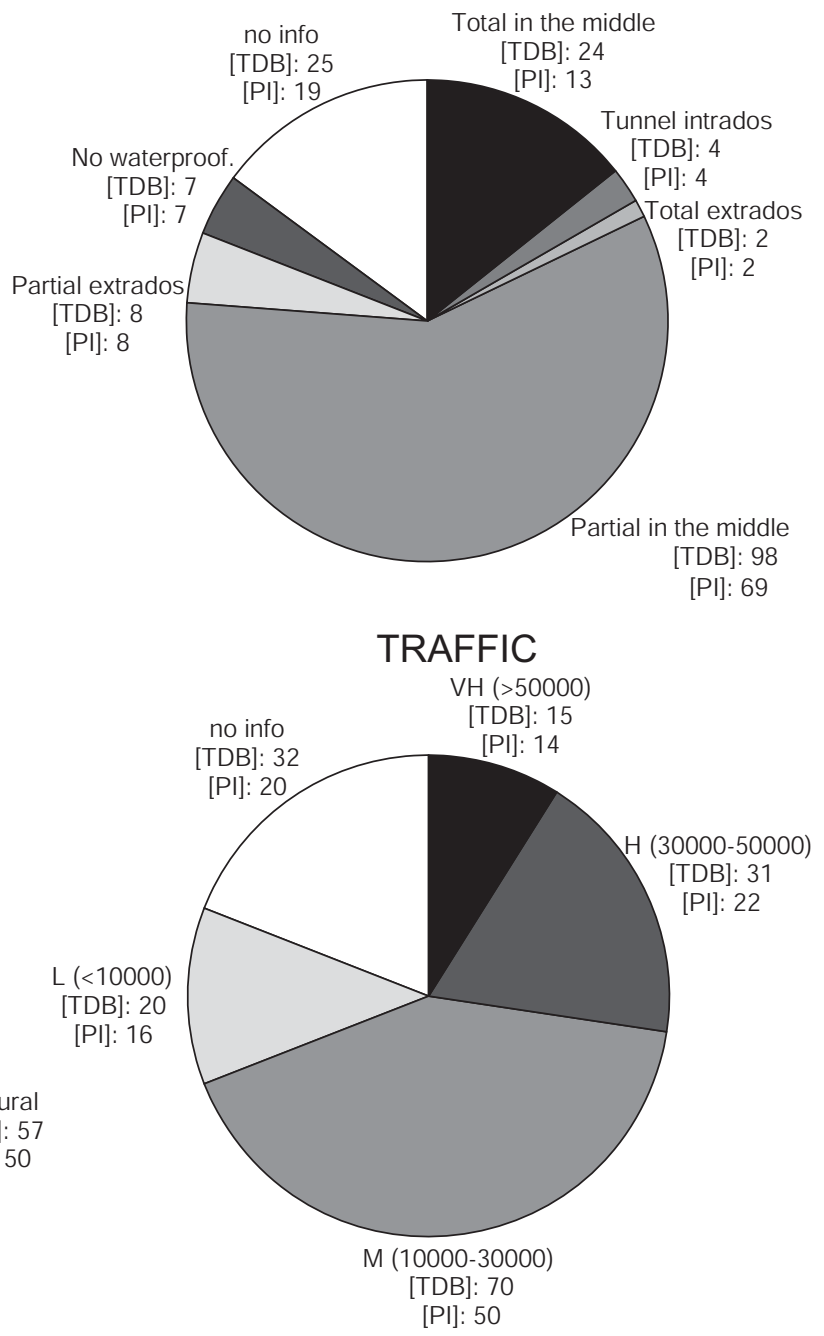

FIGURE 4. Swiss National Roads tunnels portrait. For each attribute (modality) represented in the pie chart it is specified: [TDB] the number of tunnels recorded in the TDB and [PI] the number of inspected tunnels (i.e. for which there is information about the last principal inspection). The average Daily Traffic Volume is expressed in (vehicles/day). 


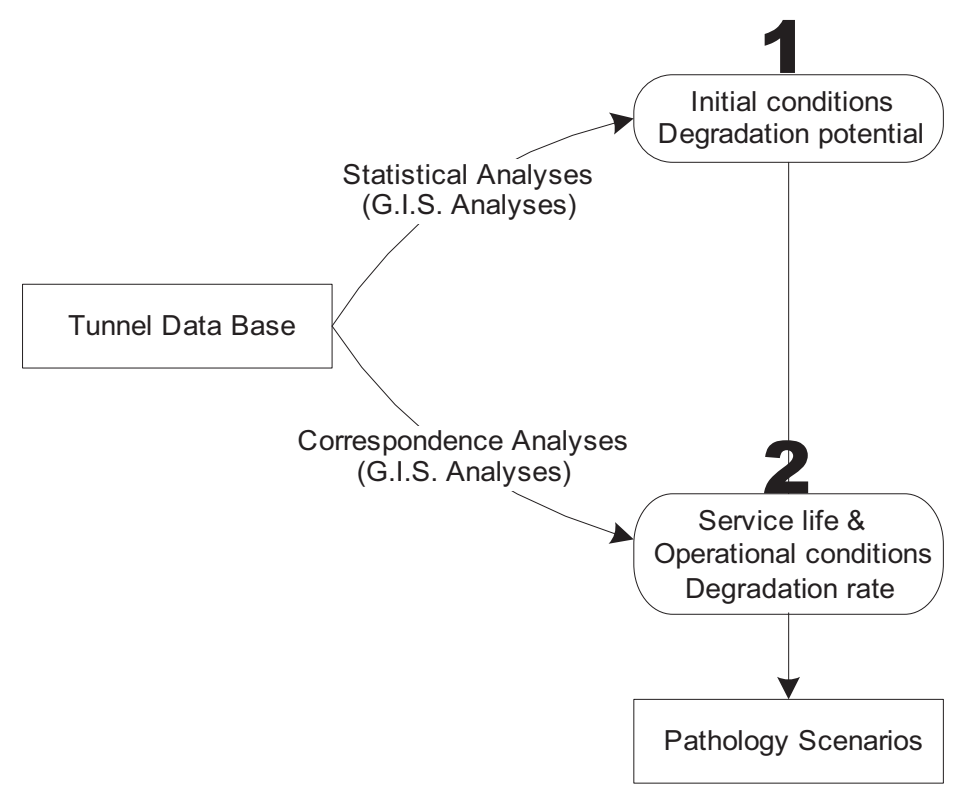

FIGURE 5. TDB Analysis process: 1. Initial conditions for investigating degradation potential and 2. Service life conditions for investigating degradation rate. The arrays describe the data analysis methods. G.I.S. analyses are between brackets since they are not presented in this paper (Sandrone et al., 2007b; Sandrone, 2008).
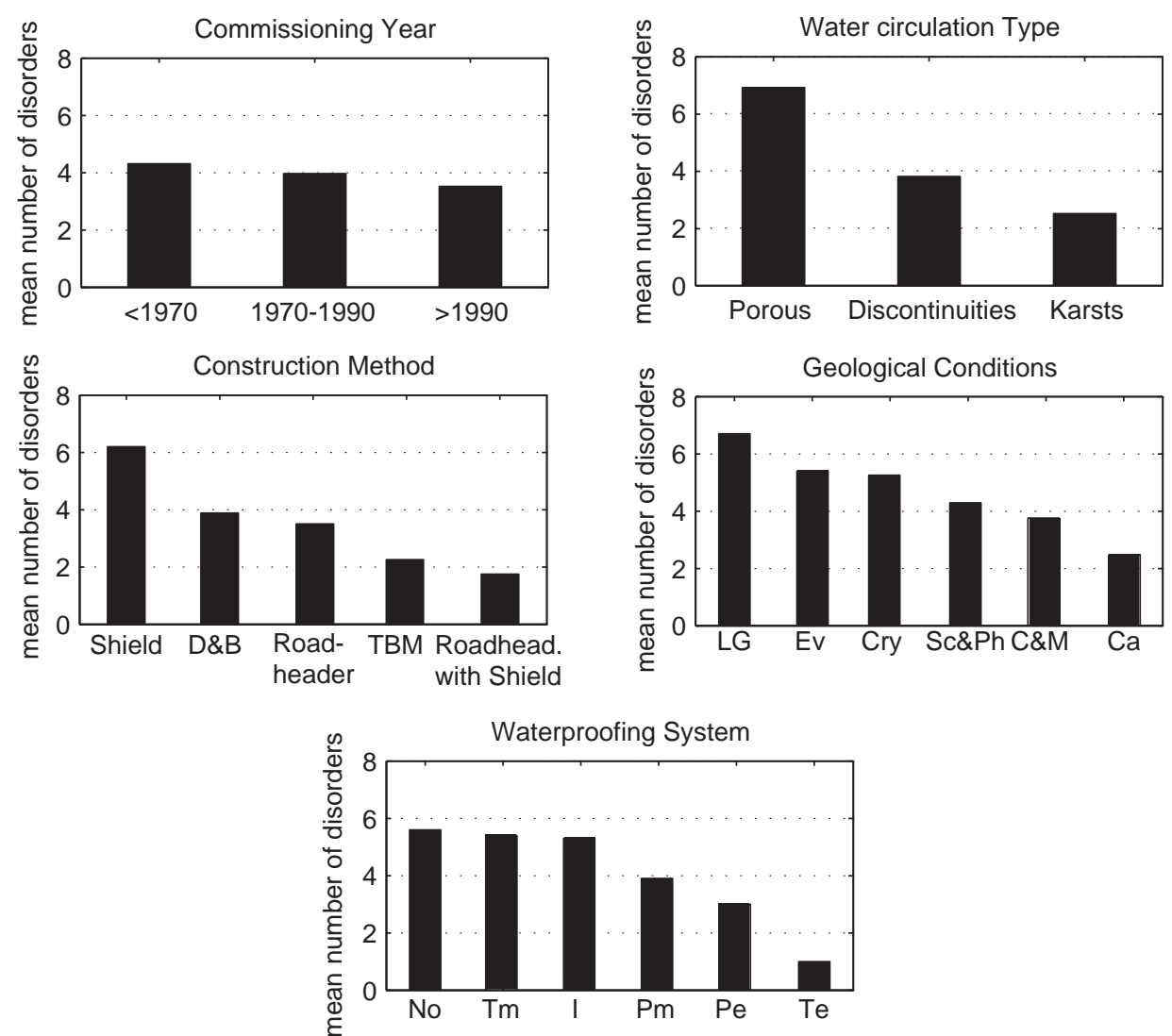

FIGURE 6. Initial conditions influence on degradation potential. For each factor it is represented the mean number of disorders per tunnel per attribute (modality) class. Geological conditions and waterproofing system modalities are described in Section 2.2 (i.e. LG = Loose Ground; Ev = Evaporites; Cry = Crystalline rocks; Sc\&Ph $=$ Schists and Phyllites; C\&M = Clays and Marls; Ca = Calcareous rocks; No = no waterproofing system; Tm = Total in the middle; $\mathbf{I}=$ Intrados; $\mathbf{P m}=$ Partial in the middle; Pe = Partial extrados; Te $=$ Total extrados). 

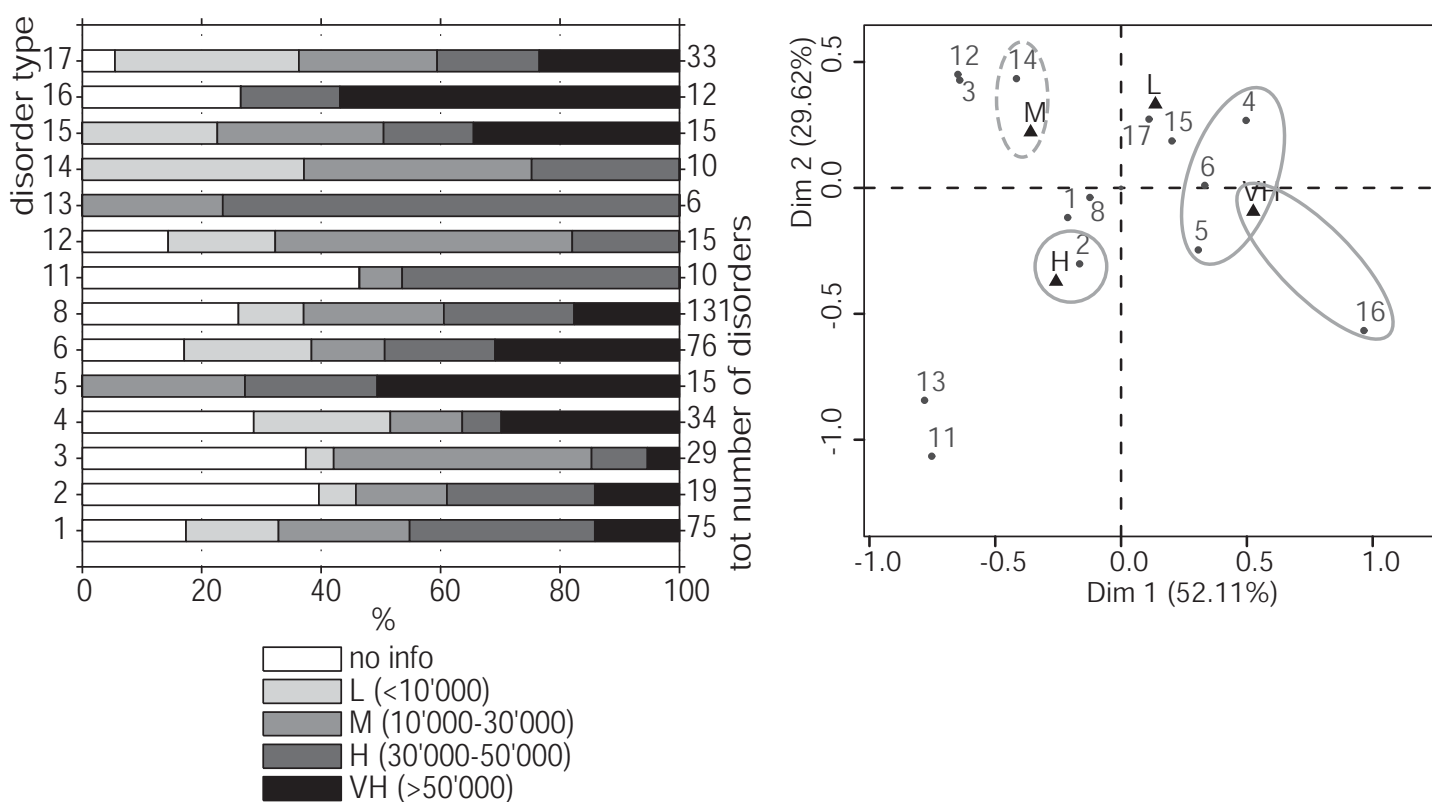

FIGURE 7. Results of the Correspondence Analysis for the average daily traffic volume, DTV (data source: OFROU, 2006). On the right a bi-plot showing similarities within disorders (numbered dots) and dependencies on influence factors (lettered triangles). Disorders list is provided in Table 3.

1.

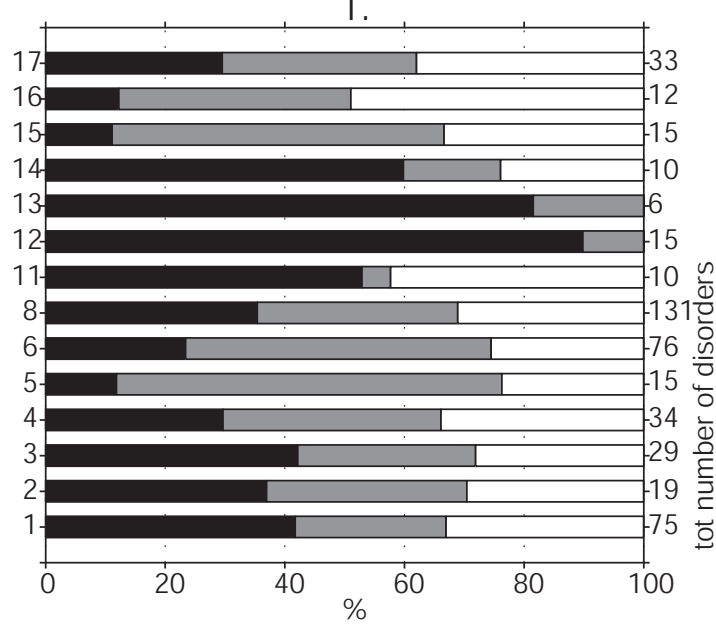

( $>40$ years) (20-40 years) ( $<20$ years)

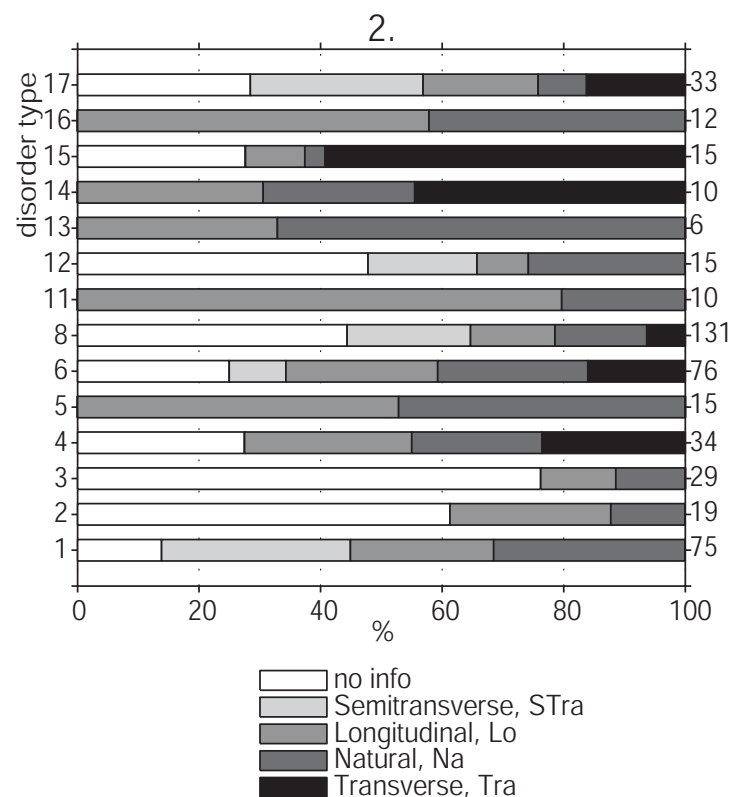

FIGURE 8. Correspondence Analysis results: 1. Tunnel age (i.e. Service life duration, calculated from the commissioning year). 2. Tunnel ventilation system. Disorders list is provided in Table 3. 
1.

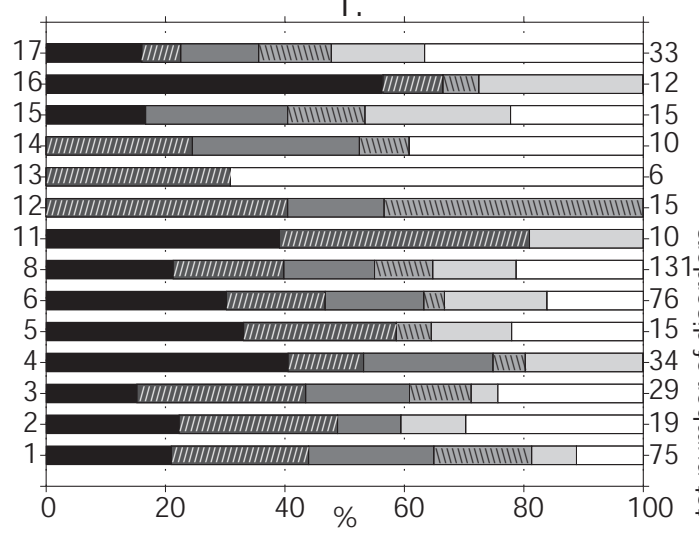

2.

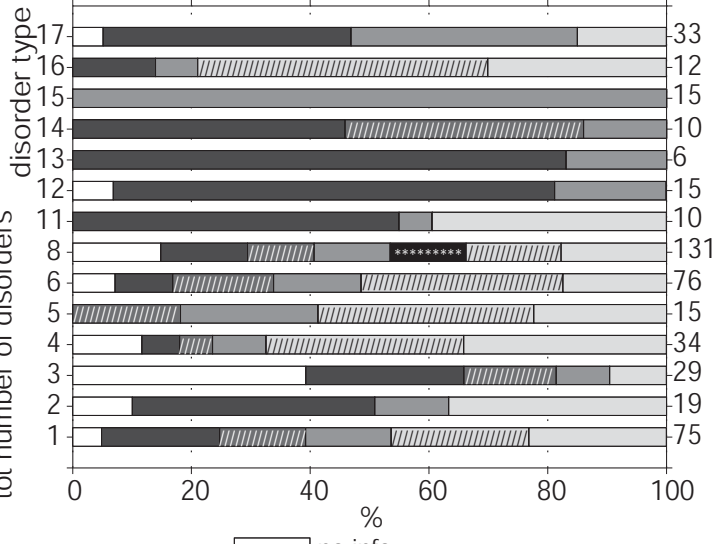

no info

No waterproofing, No

[IIIIIIIIII Partial extrados, Pe

Partial in the middle, Pm

******** Total extrados, Te

[IIIIIIIIIII Tunnel intrados, I

3.

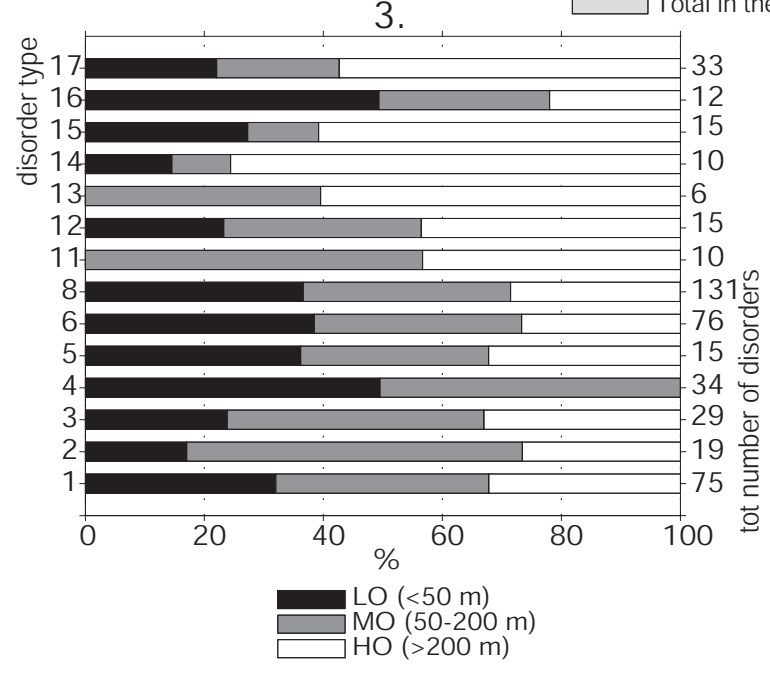

FIGURE 9. Correspondence Analysis results. 1. Tunnel geological conditions. 2. Tunnel waterproofing system. 3 . Tunnel depth. Disorders list is provided in Table 3. 
List of Tables:

TABLE 1. Principal causes of road tunnel deterioration as identified by tunnel inspectors from several countries. The sources codes are detailed as references in Section 7.1.

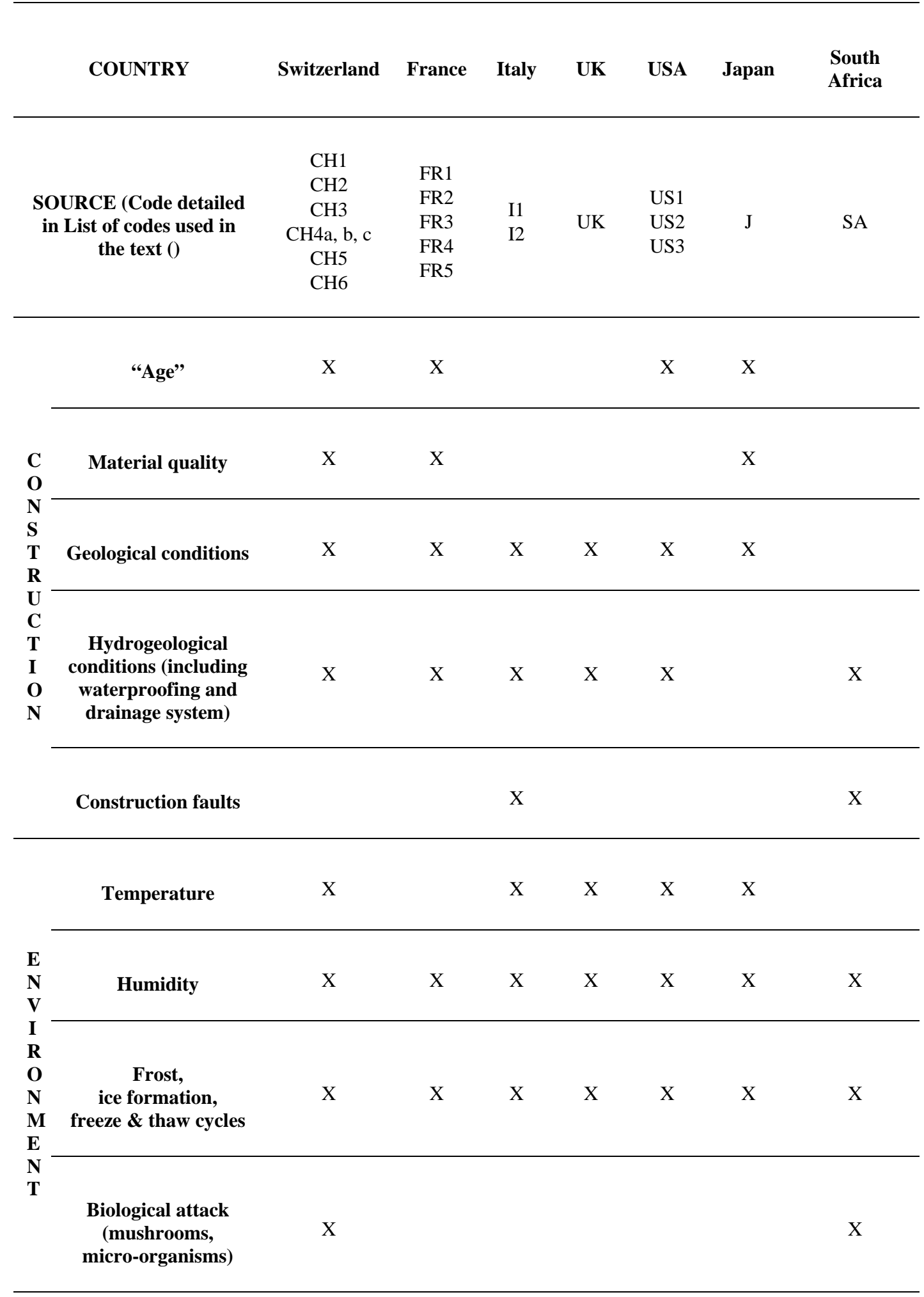




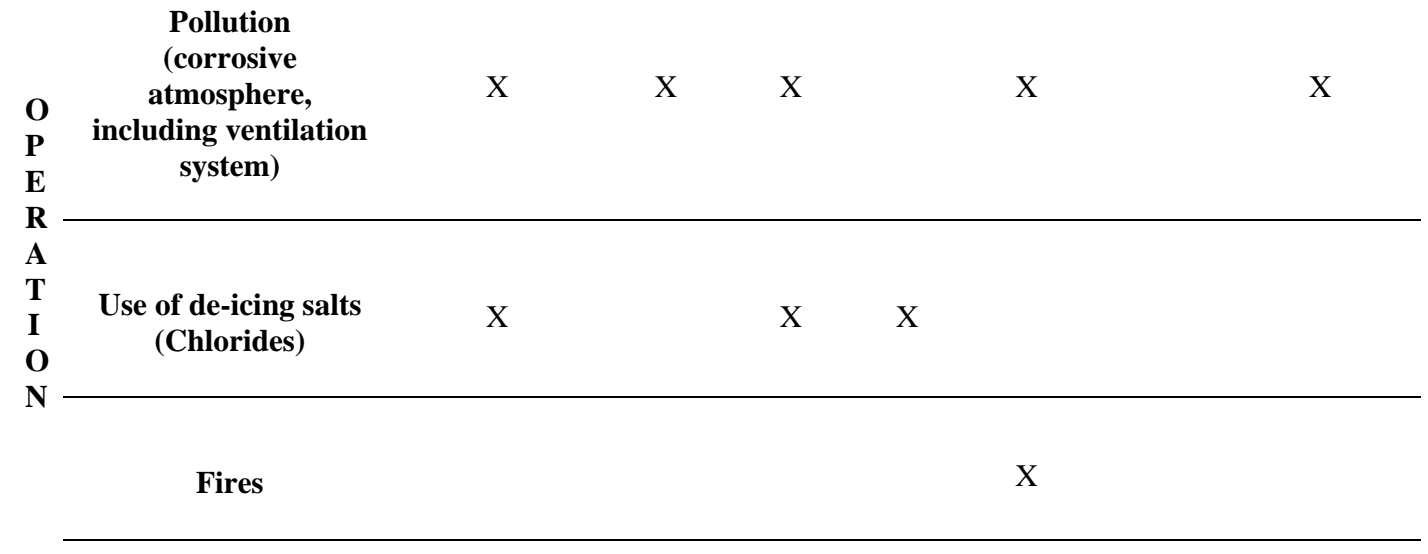

\section{Accidents}

TABLE 2. Swiss Tunnel Data Base Structure (after Sandrone et al., 2007a).

\begin{tabular}{ll} 
Section & Data \\
\hline General Information & Tunnel name \\
& Town, Canton \\
& Road \\
& Local Operator \\
& Commissioning (operation) year \\
& Coordinates X, Y (centre point) \\
& Lane Number \\
& Construction Year \\
& Geometrical Data (depth, length, section size, interaxis) \\
& Excavation method \\
& First support (type and length along the tunnel) \\
\hline Construction Information & Definitive lining (type, thickness and length) \\
& Waterproofing and drainage \\
& Accidents during construction \\
& Geological profile and description \\
\hline & Geological difficulties during excavation \\
\hline
\end{tabular}


Data

Environment and Operation Information

Accidents during operation

Traffic

Temperature

Humidity

Chemical composition of tunnel atmosphere

Chemical composition of groundwater

Groundwater level and circulation type

Technical equipment (ventilation)

Maintenance Information Inspection (date and frequency)

Monitoring

Routine maintenance

Disorders (date of observation, possible cause, area and eventual repair)

Renewal /Refurbishment (intervention date and type, area, cause) 
TABLE 3. Typical Swiss road tunnels disorders (after Sandrone et al., 2007a). For each disorder, the table shows the number of total records and of affected tunnels

\begin{tabular}{|c|c|c|c|}
\hline Type & Disorder & $\begin{array}{l}\text { Number of } \\
\text { records }\end{array}$ & $\begin{array}{l}\text { Number of } \\
\text { affected } \\
\text { tunnels }\end{array}$ \\
\hline [1] & Water leakage, Moisture & 75 & 57 \\
\hline [2] & Efflorescence (sulphates) & 19 & 16 \\
\hline [3] & $\begin{array}{l}\text { Staining, Calcium leaching effects, Calcareous concretion, } \\
\text { Honeycomb }\end{array}$ & 29 & 25 \\
\hline$[4]$ & $\begin{array}{l}\text { Concrete spalling, Delaminated concrete (due to corrosion of } \\
\text { reinforcements) }\end{array}$ & 34 & 28 \\
\hline$[5]$ & Plain concrete corrosion, Concrete scaling (de-icing salts attack) & 15 & 14 \\
\hline$[6]$ & Corrosion of steel bars (reinforced concrete) & 76 & 52 \\
\hline [7] & Voids behind the lining & - & - \\
\hline$[8]$ & Cracks, Fissures & 131 & 80 \\
\hline$[9]$ & Local deformation (crown) & 1 & 1 \\
\hline$[10]$ & Local deformation (walls) & - & - \\
\hline$[11]$ & Fines transport in the drainage system & 10 & 8 \\
\hline$[12]$ & Ice formation & 15 & 15 \\
\hline$[13]$ & Concrete lining crumbling - local failure, fragments fall & 6 & 5 \\
\hline$[14]$ & Track scaling & 10 & 8 \\
\hline$[15]$ & Invert heave up & 15 & 12 \\
\hline$[16]$ & Impact damages & 12 & 10 \\
\hline [17] & Drainage system obstruction by calcareous concretion & 33 & 30 \\
\hline
\end{tabular}


TABLE 4. Synthesis of the factors influencing the tunnel degradation rate: for each influence factor it is indicated the list of dependent disorders. The question mark '?' indicates influence factors for which no evidence has been found with data analysis but which are selected according specific literature. The disorders list is provided in Table 3.

\begin{tabular}{|c|c|c|c|c|c|c|c|c|c|c|c|c|c|c|}
\hline Influence Factor & [1] & [2] & [3] & [4] & [5] & [6] & [8] & [11] & [12] & [13] & [14] & [15] & [16] & [17] \\
\hline Age & & & & & & & & $\mathrm{X}$ & $\mathrm{X}$ & $\mathrm{X}$ & $\mathrm{X}$ & & & \\
\hline Traffic & & $\mathrm{X}$ & & $\mathrm{X}$ & $\mathrm{X}$ & $\mathrm{X}$ & & & & $\mathrm{X}$ & & & $\mathrm{X}$ & \\
\hline $\begin{array}{l}\text { Ventilation } \\
\text { system }\end{array}$ & & $\mathrm{X}$ & $\mathrm{X}$ & & $\mathrm{X}$ & & & & & $\mathrm{X}$ & & & & \\
\hline $\begin{array}{l}\text { Geology } \\
\text { Hydrogeology }\end{array}$ & $?$ & $\mathrm{X}$ & ? & & & & & $\mathrm{X}$ & $\mathrm{X}$ & $\mathrm{X}$ & & ? & & $\mathrm{X}$ \\
\hline Depth & $?$ & & & & & & $?$ & & & & $\mathrm{X}$ & $\mathrm{X}$ & & \\
\hline $\begin{array}{l}\text { Waterproofing } \\
\text { system }\end{array}$ & $?$ & $\mathrm{X}$ & $\mathrm{X}$ & $\mathrm{X}$ & $\mathrm{X}$ & $\mathrm{X}$ & & $\mathrm{X}$ & $\mathrm{X}$ & $\mathrm{X}$ & & & & $\mathrm{X}$ \\
\hline
\end{tabular}


TABLE 5. Conditional probability of disorder occurrence, given each factor modality class. The description of the class modalities is given in Section 2.2.

\begin{tabular}{|c|l|}
\hline$>45 \%$ & $\begin{array}{l}\text { Significant } \\
\text { influence }\end{array}$ \\
\hline $30 \%$ to $45 \%$ & Medium influence \\
\hline $15 \%$ to $30 \%$ & Slight influence \\
\hline$<15 \%$ & No influence \\
\hline
\end{tabular}


Influence Factor Modality [1] [2] [3] [4] [5] [6] [8] [11] [12] [13] [14] [15] [16] [17]

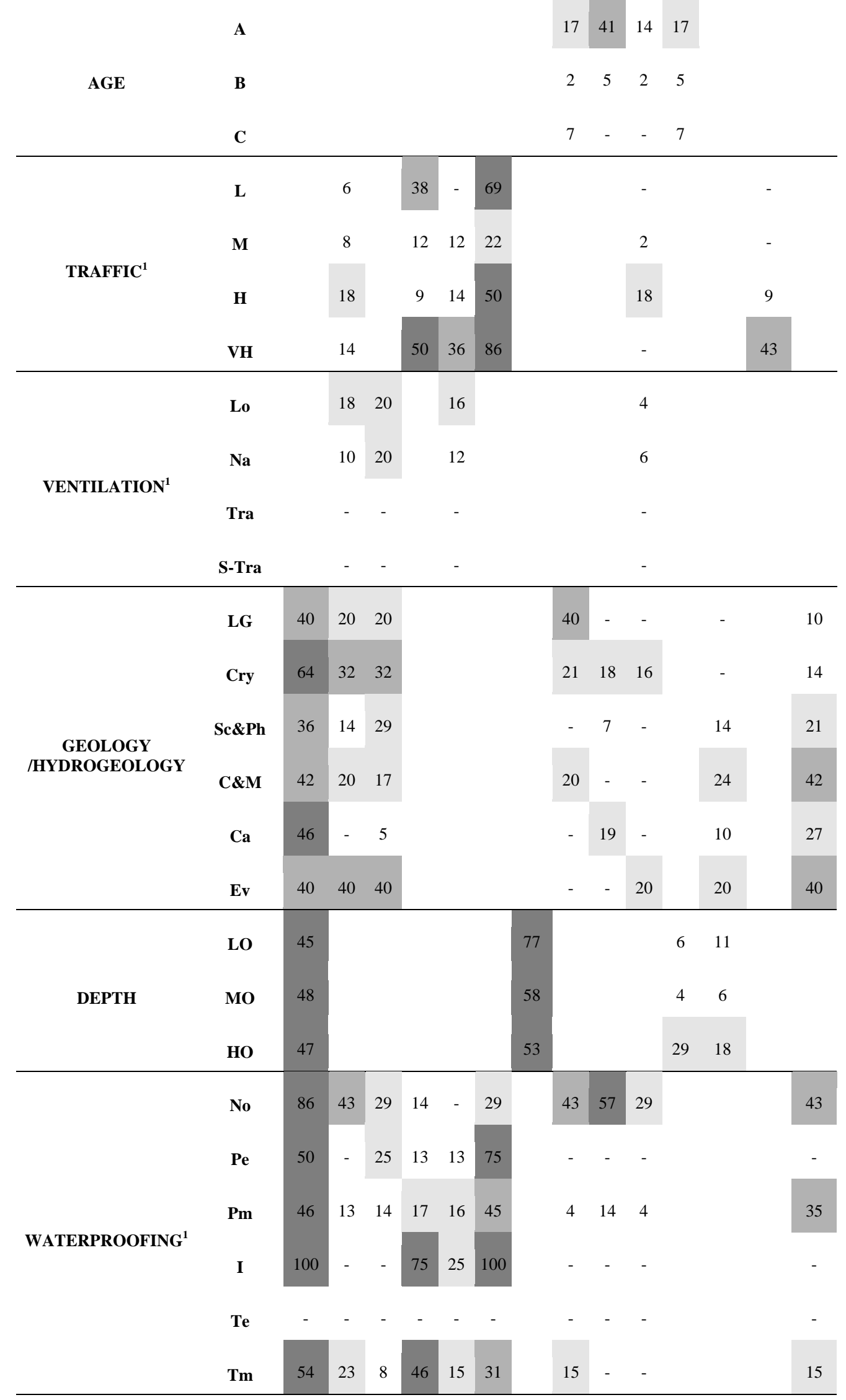

\footnotetext{
${ }^{1}$ Some of the tunnels showing the selected disorders could not be considered in this last step of the analysis process due to a lack of information on the influence factor modalities (see Figure 4).
} 